1986

\title{
Auditing, Artificial Intelligence and Expert Systems
}

Andrew D. Bailey

Rayman D. Meservy

Brigham Young University

Gordon L. Duke

Paul E. Johnson

William Thompson

Follow this and additional works at: https://scholarsarchive.byu.edu/facpub

Part of the Management Information Systems Commons

\section{BYU ScholarsArchive Citation}

Bailey, Andrew D.; Meservy, Rayman D.; Duke, Gordon L.; Johnson, Paul E.; and Thompson, William, "Auditing, Artificial Intelligence and Expert Systems" (1986). Faculty Publications. 3246.

https://scholarsarchive.byu.edu/facpub/3246

This Peer-Reviewed Article is brought to you for free and open access by BYU ScholarsArchive. It has been accepted for inclusion in Faculty Publications by an authorized administrator of BYU ScholarsArchive. For more information, please contact ellen_amatangelo@byu.edu. 


\title{
10. AUDITING, ARTIFICIAL INTELLIGENCE AND EXPERT SYSTEMS*
}

\author{
Andrew D. Bailey, Jr. \\ Academic Faculty of Accounting \\ and Management Information Systems \\ The Ohio State University \\ Columbus, Ohio 43210-1399, U.S.A. \\ Rayman D. Meservy \\ Accounting and Information Systems \\ Carnegie-Mellon University \\ Pittsburgh, PA 15213, U.S.A.
}

Gordon L. Duke, Paul E. Johnson and William Thompson

University of Minnesota

Minneapolis, Minnesota 55455, U.S.A.

1. INTRODUCTION

This paper will provide the reader with an introduction to the field of financial auditing and the applicability of Decision Support Systems (DSS), Artificial Intelligence (AI), and Expert Systems (ES) to that field of endeavor. The paper will also discuss a continuing research project concerning the application of DSS/AI/ES techniques to the evaluation of internal accounting controls. The reader will find that the evaluation of internal accounting controls is a critical step in every financial audit and that it is an area in which the auditor exhibits substantial expertise. It is thus an area of work particularly suited to the application of expert systems technology.

The current research group members each bring special and necessary inputs to the project and in some sense represent our attitude toward work in these areas. We have representatives from the functional areas of accounting and auditing, psychology and computer science. We believe that this interdisciplinary approach is the only way to assure that relevant questions are addressed within the context of the most current research and practice results from the underlying disciplines.

* We would like to thank the public accounting firm of Peat, Marwick, Mitchell \& Co., the Mcknight Foundation, and the Artificial Intelligence Research Center of the University of Minnesota for their support. The paper represents the authors' beliefs and not those of the organizations noted above. 
The specific issue addressed by the current research concerns the auditors' evaluation of internal accounting controls. Part of the current research team has been involved with the question for some time. An earlier project reported at an earlier NATO conference worked under the acronym, TICOM, or The Internal Control Model. TICOM is a computer supported description and evaluation system for accounting internal controls. While TICOM is based on AI concepts, it does not act as an ES, but rather as a DSS, providing basic information about the internal controls, but leaving all judgements to the auditor [Bailey et al., 1983].

The current research concentrates on the complete process of developing an expert system, but focuses most heavily on the cognitive science issues. Various methods of assessing the auditors' judgement processes are used in order to build the knowledge base. An expert system shell known as GALEN is used to build a computational model of the resulting process. GALEN was developed at the University of Minnesota by two of the authors [Moen, 1984]. The resulting computational model will then be validated using several validation approaches. While this research is a direct outgrowth of the previous TICOM project, TICOM does not form an integral part of this study, as this study concentrates on the cognitive aspects of the auditors'expertise. TICOM will be introduced at a later date as part of the knowledge base of the resulting computational model when the project enters its field development stage.

Our results to date are very encouraging. We are confident that the auditors do exhibit the necessary expertise in making internal control judgements and that that expertise can be represented in a computational model. This paper will present the nature of our research effort, the resulting model structure and the results obtained to date. A complete computational model is not yet available.

The rest of this paper is composed of five sections. Section 2 presents the structure of the financial auditing function and the potential aspects in which DSS/AI/ES techniques may contributt to that function. Section 3 discusses the extant research relevant in extending the expert systems work to auditing and 
accounting. Section 4 details the current research effort in understanding the auditors'judgements processes in evaluating internal accounting controls for auditing purposes and in building a computational model of that process. Section 5 presents the preliminary results of that effort and Section 6 concludes with suggestions for future research efforts in auditing and expert systems.

\section{THE AUDITING FUNCTION AND THE APPLICABILITY OF DSS/AI/ES}

This section will introduce the reader to the basic definitions, objectives and structure of the financial audit. We assume a low level of specific knowledge about the audit process when making this presentation. For those readers familiar with the topic, we ask your patience. We believe that to appreciate the need for DSS/AI/ES techniques in the auditing context and more particularly to understand the importance of the internal accounting controls issues, the reader must have a sense of the full audit context. We will attempt to point out likely application areas for DSS/AI/ES techniques as we present this material, but will expand on these points in the next section.

While accounting and auditing are related, they are distinctly different activities. "The primary purpose [of accounting] is 'to provide quantitative information primarily financial in nature, about economic entities that is intended to be useful in making economic decisions.' As an information system it captures, processes, and disseminates both externally and internally, economic information about the organization to an assortment of individuals..." Internal control issues are of significance to accountants in order to assure the accuracy, reliability and timeliness of the resulting reports and to conform to existing legal requirements such as specified by the Foreign Corrupt Practices Act (FCPA) of 1977. Internal controls are intended in this context to provide for the keeping of books, records and accounts, which in reasonable detail, accurately and fairly reflect the transactions of the assets of the organization. This is accomplished by devising and maintaining a system of internal control sufficient to provide reasonable assurance that: (a) transactiors 
are executed in accordance with management's general and specific authorizations; (b) transactions are recorded as necessary to permit preparation of financial statements in conformity with generally accepted accounting principles or other criteria applicable to such statements, and to maintain accountability for assets; (c) access to assets is permitted only in accordance with management's general or specific authorization; and, (d) the recorded accountability for assets is compared with existing assets at reasonable intervals and appropriate action is taken with respect to differences [American Institute of Certified Public Accountants, 1972]. In summary, accounting is involved with the maintenance of a system capable of preparing accurate, reliable and timely financial reports.

Auditing is a systematic process of objectively obtaining and evaluating evidence regarding assertions about economic actions and events to ascertain the degree of correspondence between those assertions and established criteria and communicating the results to interested users. This definition clearly distinguishes the accounting responsibility for the design and maintenance of systems of accounting from the function of an auditor. The auditor in this context attempts to obtain evidence in support of the appropriateness of the design, maintenance and resulting reports produced by the accountants system. The financial audit is generally considered to be an attestation audit designed to communicate a statement of opinion (judgement), based upon convincing evidence, by an independent, competent, authoritative person, concerning the degree of correspondence in all material respects of accounting information communicated by an entity (individual, firm, or governmental unit) with established criteria.

The problem of established criteria is not a trivial one for either accountants or auditors. The criteria for the financial reports are established by the Financial Accounting Standards Board (FASB) subject to oversight by the Securities and Exchange Commission (SEC), the Court system and established practice. The established criteria for the system designed to produce these reports is less well established. In general, accepted practice 
augmented by limited analysis has been the audit criteria. The Statements on Auditing Standards provide broad guidelines for the implementation of control systems. The following categories of concerns are enumerated:

1. Establishment and supervision of internal accounting control systems is a management responsibility.

2. Absolute assurance of effectiveness is probably not cost effective; thus, reasonable assurance is acceptable.

3. Concepts of internal control are independent of the data processing mechanism. (This applies to computer processing as well as manual processing of transactions.)

4. Any system of control may be compromised by error, collusion, management override, or deterioration in compliance.

5. Competent personnel of high integrity are essential to good internal control.

6. Segregation of functions implies that those in a position to perpetrate "error" should not also perform functions enabling them to conceal those "errors." For instance, those who control assets should not also control the accounting for assets.

7. There is a need to generate independent evidence supporting valid authorization, approval, and performance of actions.

8. Proper documentation, recording, authorization, and approval of transactions must be maintained.

9. Access to assets must be limited to authorized personnel.

10. Periodic comparisons of recorded amounts to actual assets and follow up on deviations are essential to good internal control.

Based on broad guidelines of this nature as to the system designed to capture, process and manipulate economic data for the preparation of financial reports consistent with generally accepted accounting principles of reporting, auditors attempt to gather evidence about managements reports.

The evidence gathering process itself can be organized for descriptive purposes into four basic phases as illustrated in Figure 1: Planning and Design of the Audit Approach; Tests of 


\section{GATHERING AUDIT EVIDENCE}

PHASE I

PLANNING AND

DESIGN OF AUDIT APPROACH

PHASE II

TESTS OF

TRANSACTIONS

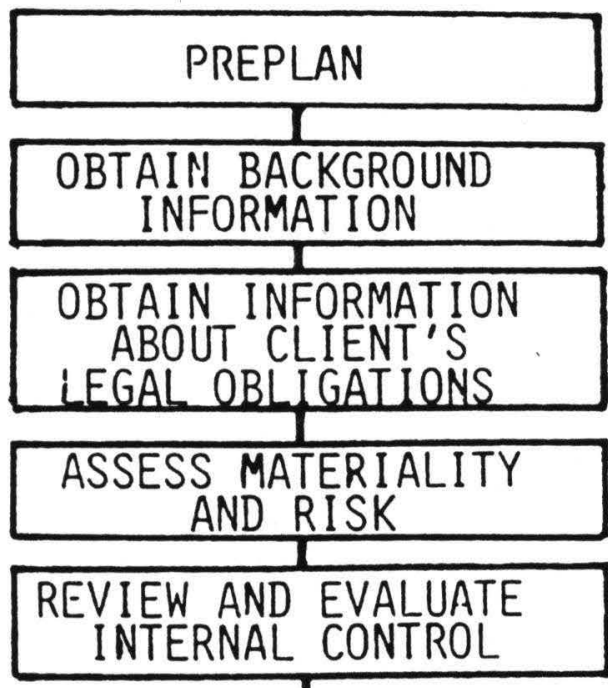

DEVELOP

OVERALL AUDIT PLAN

AND AUDIT PROGRAM

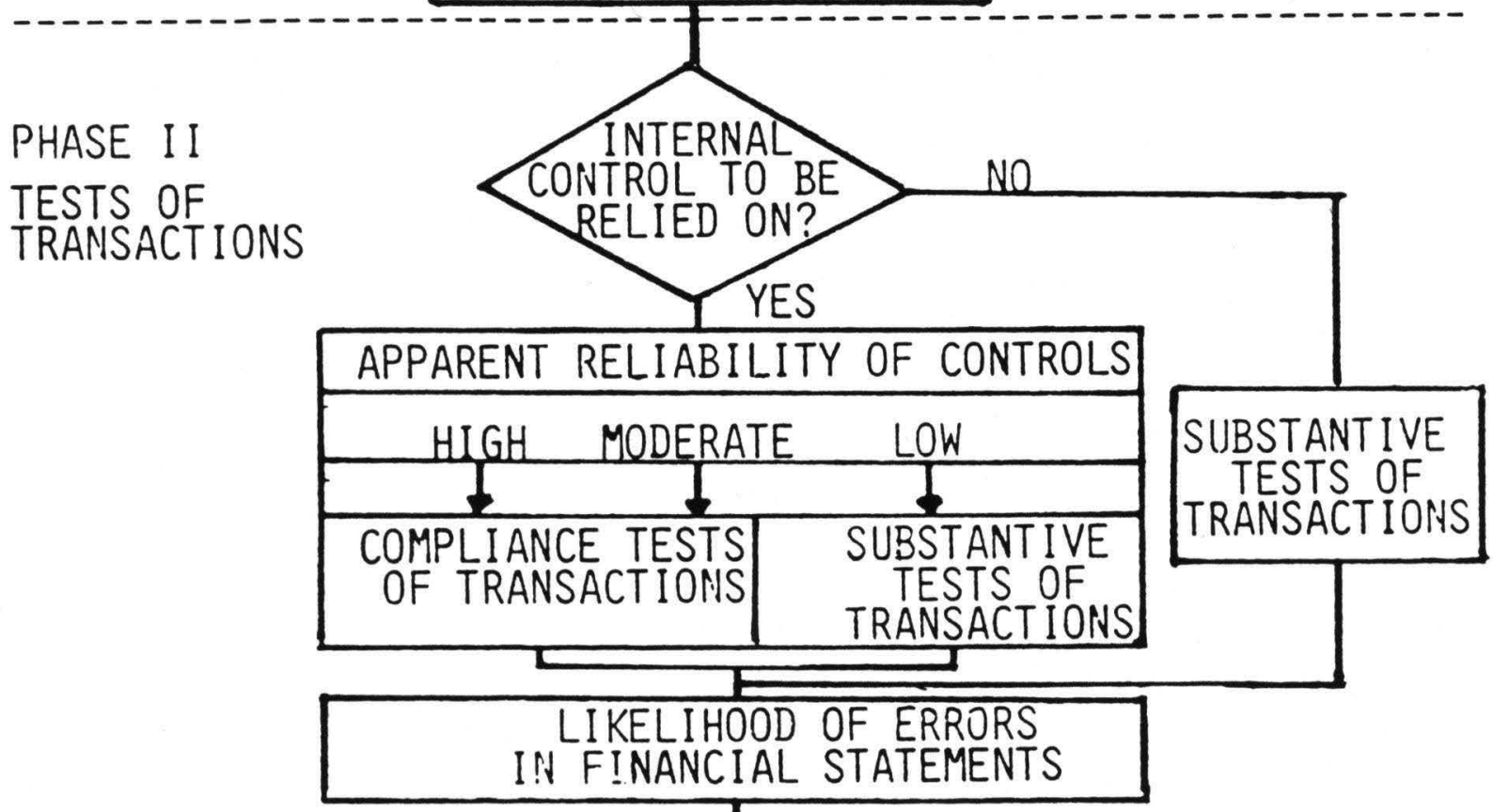

A.A. ARENS/J.K. LOEBBECKE, AUDITING: AN INTEGRATED APPROACH, 3RD ED., PRENTICE-HALL., ENGLEWOOD CLIFFS, N.J., COPYRIGHT 1984. ADAPTED FROM IATERIAL BY TOUCHE ROSS \& CO., COPYRIGHT 1978. REPRINTED BY PERMISSION. 


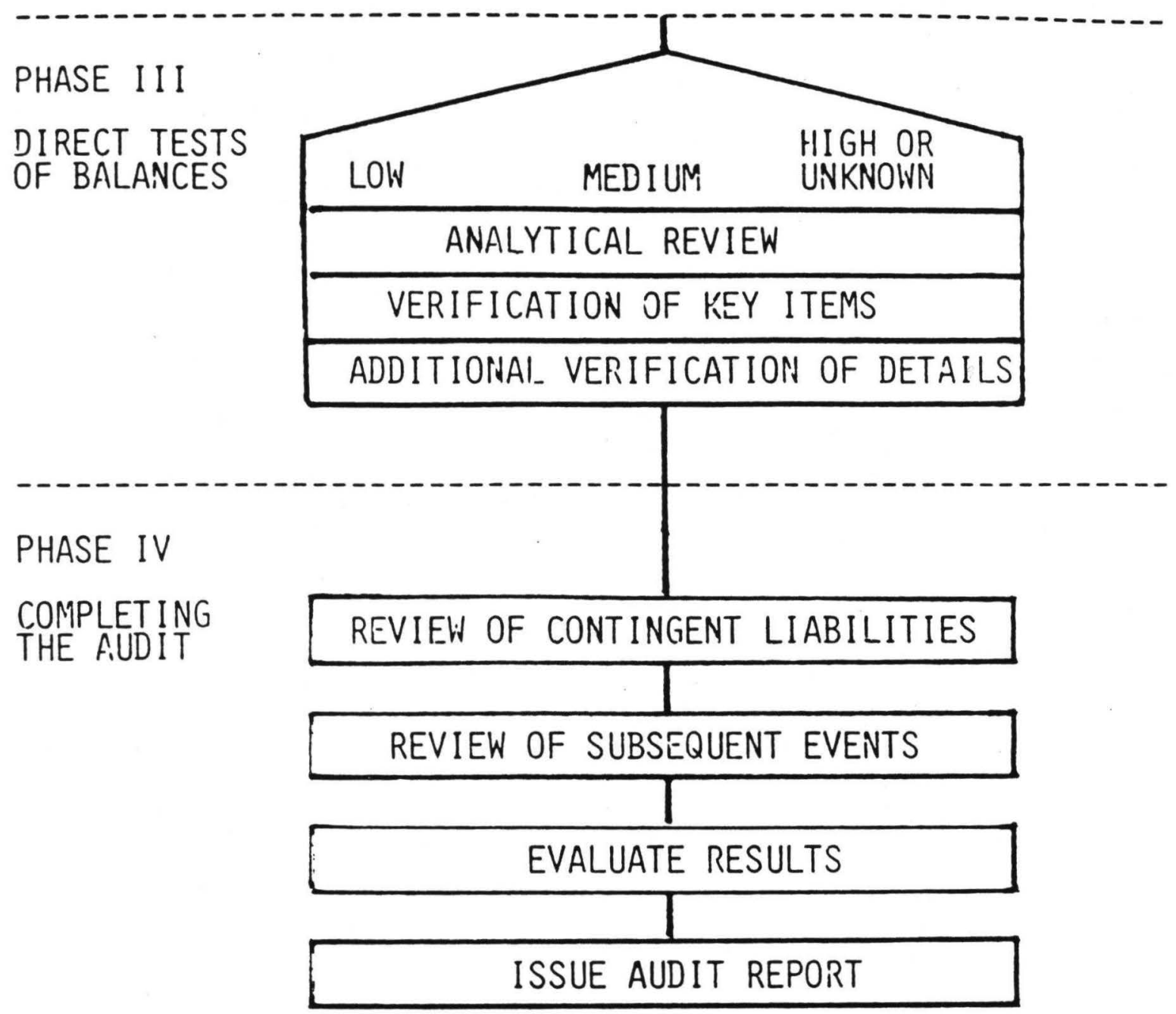

A. A. ARENS/J,K. LOEBBECKE, AUDITING: AN INTEGRATED APPROACH, 3RD ED., PRENTICE-HALL., ENGLEWOOD CLIFFS, N.J., COPYRIGHT 1384. ADAPTED FROM MATERIAL BY TOUCHE ROSS \& CO., COPYRIGHT 1978. REPRINTED BY PERMISSION.

Figure 1 (cont.) 
Transactions; Direct Tests of Balances; and, Completing the Audit [American Institute of Certified Public Accountants, 1972].

Within each of the basic four phases are substantially more detailed sets of activities and judgements necessary to operationalize the process. We also recognize that this simple lineal scheme is convenient for exposition, but that the process in fact proceeds in a much less obvious progression of events. To provid, some sense of the increasing complexity of the process consider the "Assess materiality and risk" box with the Planning Phase of the audit. Figures 2 and 3 are suggestive of the estimates and judgements necessary. Both of these processes are critical to the audit planning and implementation process and are reputed to involve substantial auditor expertise in their evaluation. Littlc objective data is available as to how auditors make these assessments or how they subsequently incorporate them into the subsequent phases of the audit.

Both of the above assessments are related to the evaluation of internal controls, which involves substantive judgements as suggested by Figure 4, part of the Planning process, and by Figure 5, part of the Tests of Transactions process.

These figures allude to the complexity of the audit task, both in terms of the number of judgements necessary and the lack of objective, normative criteria for specifying either the judgement itself or its relationship to other parts of the audit.

The applicability of DSS/AI/ES concepts in the auditing environment is self-evident. Audits are complex decision-making problems well beyond the capacity of analytic solution even if many of the judgement aspects of the audit could be reduced to simple quantitative relationships [Bailey et al., 1981]. At numerous points in an audit the auditor is called upon to exercise what can only be called expertise: assessing materiality and risk for a specific client; evaluating internal control; planning an audit based on the assessment of risk, materiality, and other inputs; revising the plan based on results obtained during the audit; and, choosing a final opinion. 


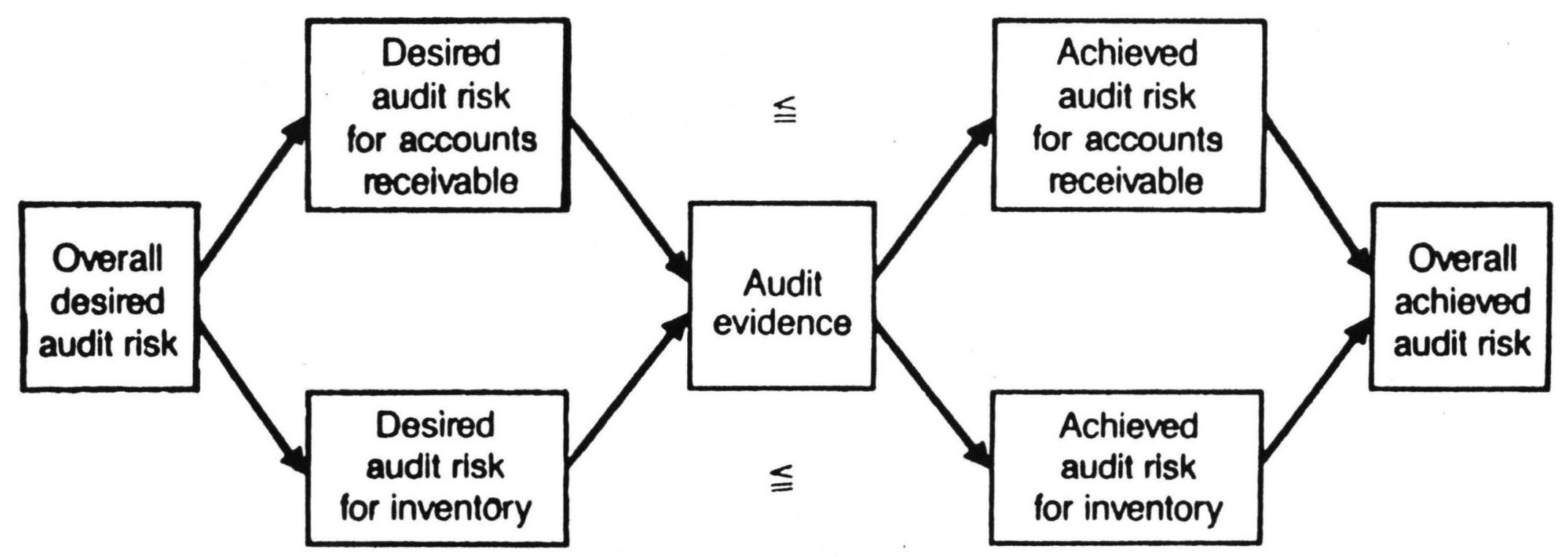

A.A. ARENS/J.K. LOEBBECKE, AUDITING: AN INTEGRATED APPROACH, 3RD ED., PRENTICE-HALL, INC., ENGLEWOOD CLIFFS, N.J. COPYRIGHT 1984. REPRESENTED BY PERMISSION.

Figure 2 


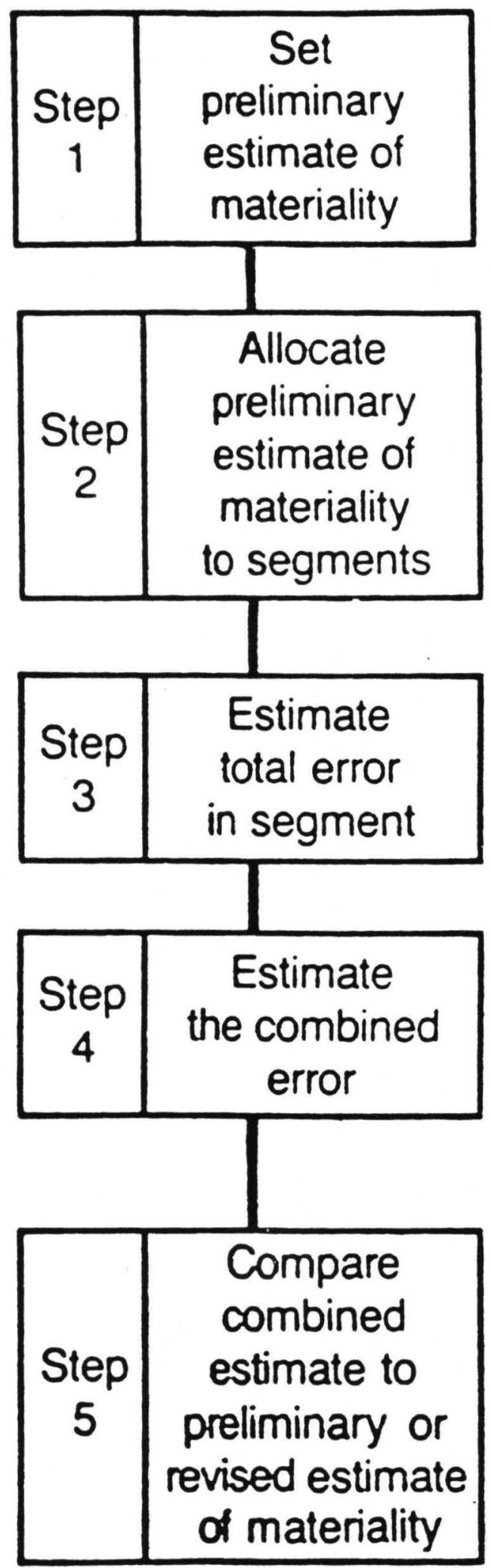

A.A. ARENS/J,K. LOEBBECKE, AUDITING: AN INTEGRATED APPROACH, 3RD ED, , PRENTICE-HALL, INC, ENGLEWOOD CLIFFS, N,J, COPYRIGHT 1984. REPRESENTED BY PERMISSION. 


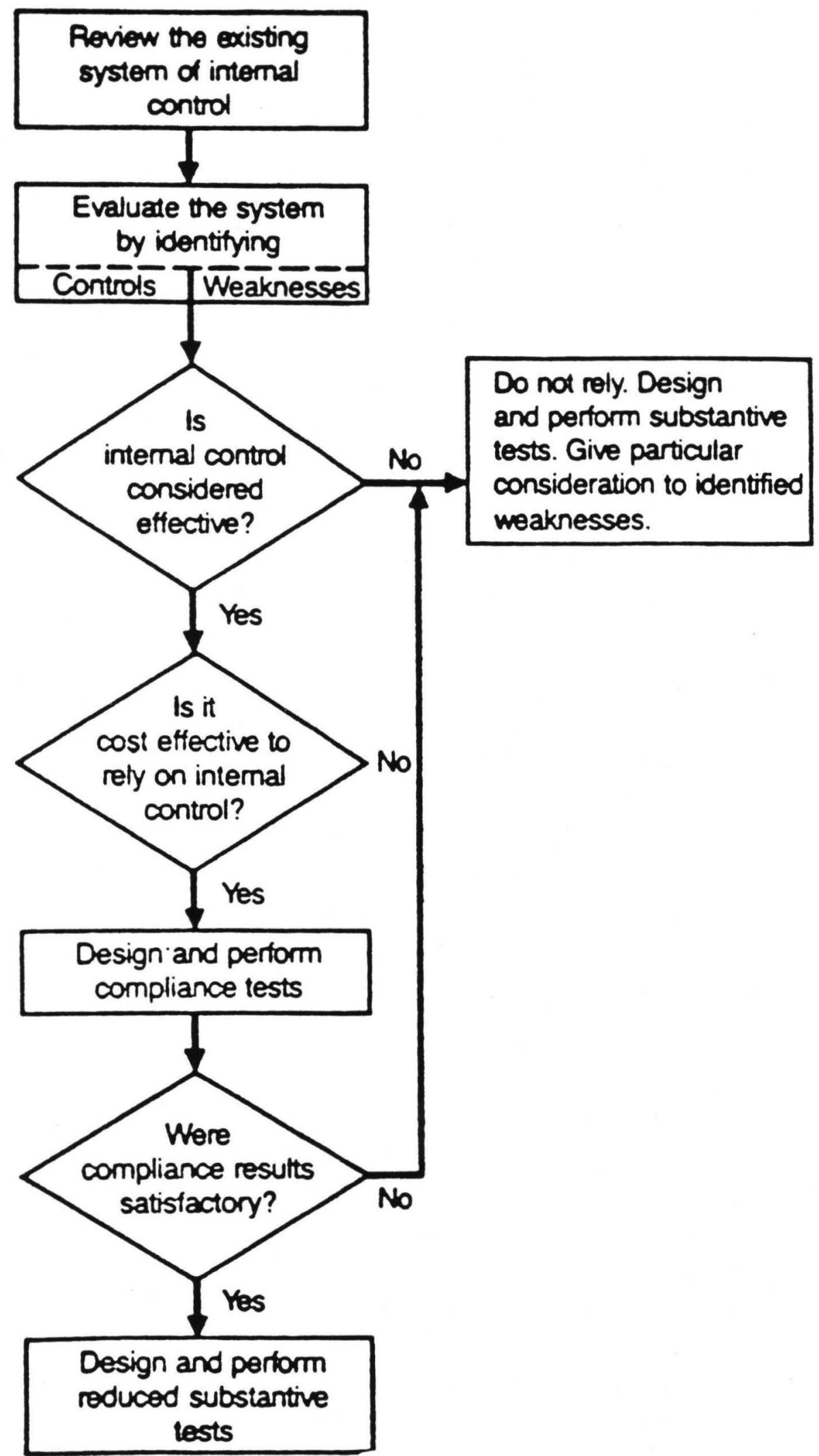

A.A. ARENS/J,K, LOEBBECKE, AUDITING: AN INTEGRATED APPROACH, 3RD ED, , PRENTICE-HALL, INC, ENGLEWDOD CLIFFS, N.J. COPYRIGHT 1984. REPRESENTED BY PERMISSION.

Figure 4 


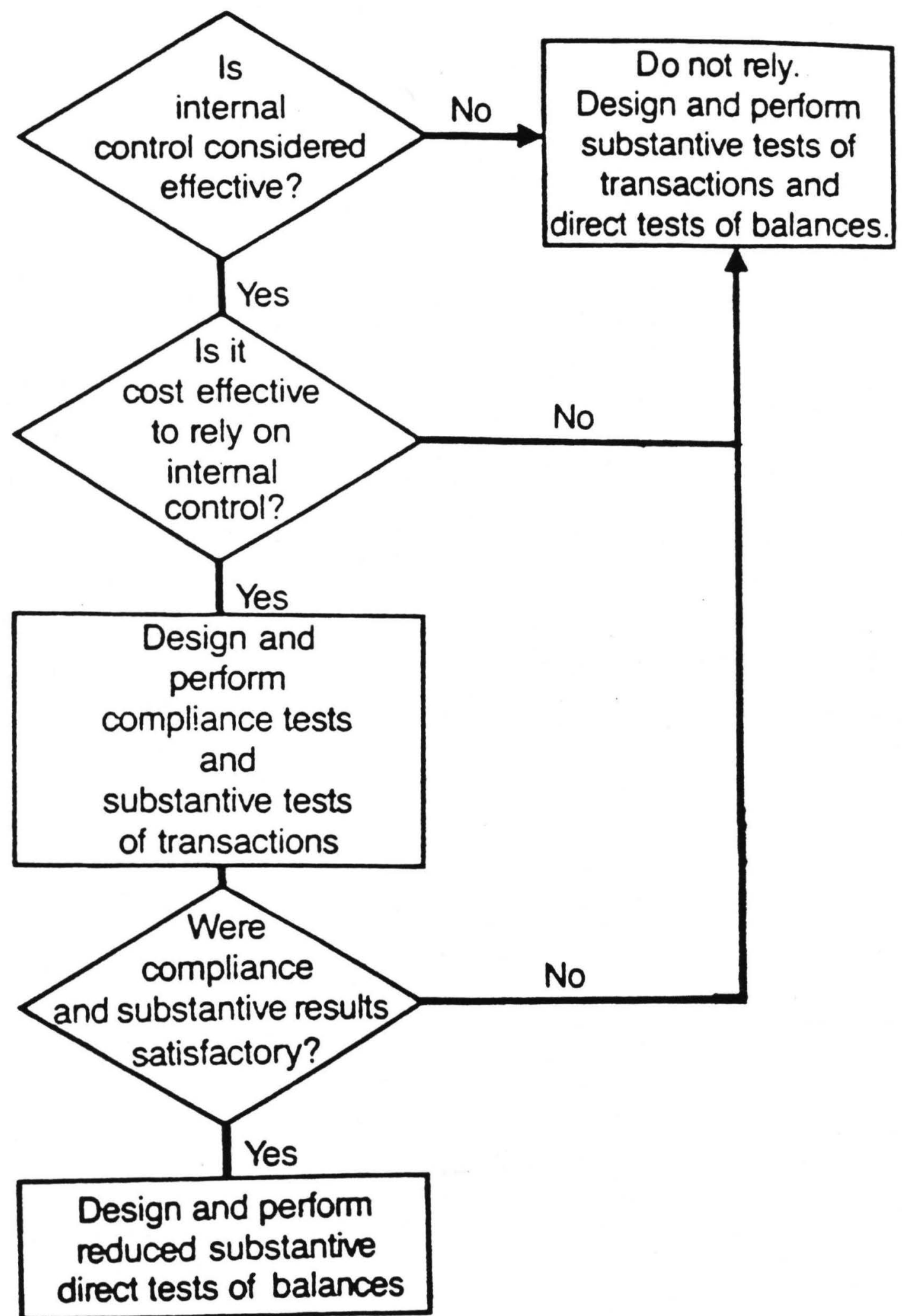

A, A, ARENS/J,K, LOEBBECKE, AUDITING: AN INTEGRATED APPROACH

3RD ED., PRENTICE-HALL, INC., ENGLEWOOD CLIFFS, N.J, COPYRIGHT 1984. REPRESENTED BY PERMISSION. 


\section{EXTANT AUDITING DSS/AI/ES APPLICATIONS}

Interest in DSS/AI/ES and other computer-based decision tools is epidemic among accountants and auditors. The Symposia on Decision Support Systems for Auditing and Accounting at the University of Southern California attests to this fact. This section briefly reviews the existing literature in auditing as it relates to DSS/AI/ES.

There are many views about what should be included as DSS and their relationship to AI and ES. While no definitive statement of definitions and characteristics for DSS exist, the following are indicative of many of the lists of such characteristics.

1. Computer Based -- It's possible to implement a DSS without a computer (certainly paper-based systems do exist), however, it is the processing power of computer technology that makes most DSS practical. All of the DSS discussed in this paper are computer-based systems.

2. Interactive -- Interactive computer usage means that the computer may interrupt processing to query the user for input. At the same time, the computer may provide information on the results of processing thus far. Another form of interaction includes a query by the user requesting additional feedback or data.

3. Data Retrieval and Manipulation -- DSS commonly support information access to large data banks. Information access may include the abilities to selectively retrieve, summarize and classify. In some DSS applications, such as data base management systems, data retrieval and manipulation is the main function.

4. Decision Model -- Many DSS applications have as their main function the implementation of a decision model. A decision model combines data and decision rules to sug,gest a course of action. A decision rule is a logical inequality that evaluates to either true or false or into some vector of results with attendant probability assignments.

In this paper, we use the term Decision Support Systems or 
DSS in a broad sense to refer to any interactive computer application that helps a decision maker by providing access to large data banks or by implementing a decision model or both.

Artificial Intelligence (AI) has been defined by Barr and Feigenbaum [1981] as "the part of computer science concerned with designing intelligent computer systems, that is, systems that exhibit the characteristics we associate with intelligence in human behavior -- understanding language, learning, reasoning, solving problems, and so on." AI systems may use normative or optimal modelling techniques or they may mimic human decisionmaking processes. Furthermore, they may or may not be designed as an aid for decision makers. A sub-classification of AI is ES. Like AI, expert systems may use optimal modelling techniques or mimic human decision-making processes, and may or may not be designed as aids for decision makers. However, most ES tend to mimic the heuristics of experts.

Expert systems have been defined by Stefik et al. [1982] as "...problem-solving programs that solve substantial problems generally conceded as being difficult and requiring expertise. They are called knowledge based because their performance depends critically on the use of facts and heuristics used by experts." Feigenbaum [1978] says, "we must hypothesize from our experience to date that the problem solving power exhibited in an intelligent agent's performance is primarily a consequence of the specialist's knowledge employed by the agent, and only very secondarily related to the generality and power of the inference method employed. Our agents must be knowledge rich, even if they are methods poor." Thus, expert systems attempt to capture specific knowledge from an acknowledged expert concerning a specific problem domain and replicate the decision inference process used by this expert. In this paper, expert systems is used in a narrow sense to refer to interactive computer applications that help a decision maker by simulating the specific knowledge and inference processes used by experts in their limited domain of expertise.

Alter [1977] proposed a taxonomy of decision support systems that covers a range from data oriented to model oriented systems. We propose a three way taxonomy based on the nature of the DSS in 
Auditing Applications. We arbitrarily divide the nature of the DSS into Decision Aids, Non-Expert DSS, and Expert Systems. A system was considered a decision aid for our purposes when it was insufficiently interactive to be classified as a Non-Expert DSS or ES, i.e., level of interaction was the distinguishing characteristic. At the other extreme an ES was distinguished from a Non-Expert DSS on the basis of the degree to which the DSS attempted to emulate the expert's decision processes rather than providing model based solutions to problems. This characterization, like each of the others, is flawed, but will serve our purposes in reviewing the auditing literature to date.

\subsection{A Review of Auditing Decision Support Systems}

Auditors have long been involved with DSS, long before the computer made its practical debut. Auditing firms are an assembly of independent professionals. In order to attain a common level of professional performance and to train new professionals in the field, auditing firms found that training programs and field decision aids could contribute substantially to improved professional judgement. The issue of measuring the improvement from an auditing context is not settled and perhaps cannot be in any definitive manner. Unlike the medical diagnosis areas where the use of ES first took hold with MYCIN [Shortliffe, 1976] or in the chemical compounds area represented by DENDRAL [Lindsay et al., 1980], in many areas of auditing there is no practical and definitive means of ascertaining the correctness of a professional judgement. In theory, an exhaustive search of the clients' records might permit an accurate assessment, but practical economics precludes such a process except very rarely. Thus the measures of success have tended to focus on professional consensus .

The development of organized methods of collecting, organizing and scoring data collected on audits was a long established practice before the computer. However, the advent of inexpensive computing has opened new horizons. As a result, virtually all of the manual aids previously used by auditors have or are being converted to computer support systems. These include the systems 
prepared by the major Public Accounting firms, as well as private software houses catering to the Public and Internal Auditing communities. Most of these systems concentrate on data collection from client systems, organization, analysis based on descriptive and normative models, statistical sampling methods, and workpaper control. They are, in most cases, Decision Aids, lacking the characteristics necessary to be a Non-Expert DSS. The list of such systems is an alphabet soup of acronyms including: ASK, AUDITAID, BASE, CARS, PROBE and numerous others [Weber, 1982].

These Decision Aids are computer based, but provide little interactive support, relying on the auditor to select from among the available models for data collective, organization and manipulation. Many of the Decision Aids come equipped with normative models for data analysis purposes, but do not provide a direct recommendation to the auditor or direct the auditor along new paths of inquiry.

In the last several years auditing firms have become very active in extending the capabilities of Decision Aids by adding menu-driven, interactive components to support the various audit functions. Peat, Marwick and Mitchell's SEACAS system is perhaps the earliest and best developed of these systems. Other NonExpert Decision support systems work in auditing includes the Balachandran and Zoltners [1981] staff scheduling model, the Mock and Vertinsky [1984] risk assessment program, and several efforts in providing training systems in Bayesian revision for auditors. These systems appear to be true Non-Expert DSS, possessing the characteristics noted above, but without effective query capability and no expert analysis potential at this time.

Non-Expert DSS are part of a new wave of technological innovation in the field of auditing. Auditors accept both the technology and the potential. Within the next several years we will see numerous such systems coming on line.

AI related research in auditing starts with the work of Bailey, Gerlach and Whinston and the TICOM II project. TICOM is the acronym for the Internal Control Model. TICOM I was not based on artificial intelligence concepts, but rather on a data based model. TICOM III [Bailey et al., 1985] is the prototype 
operating systems of TICOM II. TICOM III is a Non-Expert DSS in that, while it employs many artificial intelligence-like analysis methods, it does not provide for an expert judgement by the system itself. This is still left to the auditor. Nevertheless, in concept TICOM differs from the previous DSS efforts in its explicit attempt to provide an intelligent analysis of a system of internal control based on the auditors'criteria and supported by a query system closely approximating the natural query processes used by auditors.

In the last few years, numerous projects have been started that attempt to apply expert systems techniques, a sub-category of AI concepts, in the development of expert systems for auditors. These include the development of TAXMAN and TAX ADVISOR [Michaelson, 1982] to provide legal tax advice. TAX ADVISOR is based on the EMYCIN [van Melle et al., 1981] shell. AUDITOR [Dungan and Chandler, 1980] is an expert system for the evaluation of the adequacy of the client's allowance for bad debts. Systems under development include ones by Braun and Chandler [1982] to aid auditors in Analytic Review, by Wright at the University of Minnesota to provide an evaluation of the bank loan credit assessments, by Hansen and Messier [1982] model for evaluating EDP Controls, and by Bailey, Duke, Johnson, Meservy and Thompson [1984] for internal control evaluation. The Peat, Marwick, Mitche11 Foundation, through its Research Opportunities in Auditing (PMM/ ROA) program funded several additional projects in its most recent round of awards [Peat, Marwick, Mitchel1 \& Co., 1976].

The interest and acceleration of current activity in building DSS in auditing provides a clear indication that research and development of such systems is about to explode. The above brief discussion of systems in auditing represent only the tip of the iceberg of current interest and activity. Further, this discussion ignores the many studies necessary to the final implementation of such systems. Studies such as that of Biggs [1984] on the Gong Concern judgement process, Mutchler [1984] on the issues of Subject To opinions, and numerous others facilitate the future development of DSS for auditors.

From the discussion in Section 2, it should be clear that 
numerous expert judgements are necessary in an auditing context. Each of these judgements must be studied in order to gain an understanding of the auditor's judgement processes and then placed within the context of an appropriate computational model, one that uses an analysis methodology analogous to the auditor's. Fundamental research issues must be addressed in many of these areas before the development of expert systems or other DSS can be considered. Significant behavioral issues remain unresolved. In addition, new developments in artificial intelligence will be needed if the richness of the auditing situation is to be embedded in expert systems that are more than small fragments of the audit.

The rest of this section presents a short history of the development of our current research effort in this area. It covers a part of the TICOM research and an ongoing study.

\subsection{The Internal Control Mode1-TICOM}

TICOM is a computer-based analytic tool that aids the auditor to first model the internal control system and then to query the model in order to aid the auditor in evaluating the internal control system. In the context of our previous discussion, it is a Non-Expert Decision Support System. However, TICOM is based on concepts in artificial intelligence such as knowledge representation and graph simplification. It was developed to aid auditors in the design of new systems or the description of existing internal accounting control systems and to allow the auditor to query the description. The artificial intelligence properties of TICOM permit a limited analysis and evaluation of the system in response to the auditor's query.

TICOM is composed of two sets of interrelated programs, the Internal Control Description Language (ICDL) compiler and the query processor. TICOM is the first auditor decision support system designed to permit auditors to perform their traditional tasks of internal control description and evaluation while using the power of the computer in an artificial intelligence like mode. The ICDi allows the auditor to develop internal accounting control descriptions at any level of detail or aggregation, maintaining 
information on agents, objects, repositories and commands necessary for the description.

Agents are the actors in the system who perform a task. Individual agents or groups of agents manipulate the objects within the system. Repositories represent storage locations for objects, such as file cabinets, magnetic tapes, buildings, etc. Agents' tasks are described using one or more commands. The command list is reasonably short including: ASSIGN, MODIFY, DESTROY, TRANSFER, WAIT-FOR, PUT, GET, COPY, END-TASK, REVIEW, and the boolean IFTHEN-ELSE .

The internal representation of a TICOM system description is in the form of a bilogic directed graph showing both control and data flows. A partial ordering of the ICDL description is explicitly encoded in the internal representation. As a result, analytic procedures can operate directly on the internal representation. Analysis makes use of the concepts of contraction and simplification in order to respond to a user query.

In developing our current project, we initially intended to simply extend TICOM by adding appropriate evaluation functions based on the results of the query process. While this may seem like a natural extension to an expert system, we again encountered a difficulty which we had earlier put aside. We, in fact, did not know how the auditor arrived at an evaluation based on the results of querying the system of internal control. As a result our current efforts are directed to the cognitive aspects of the auditor's decision process in an attempt to derive the auditor employed heuristics.

TICOM is not used directly in this study. Completion of this study will, however, lead to the incorporation of TICOM as a part of the static knowledge base of any expert system dealing with the identification of internal control evaluation activity.

4. AUDITING INTERNAL CONTROLS: A COMPUTATIONAL MODEL

Researchers, as well as teachers and practitioners, are interested in the processes that experts use when making judgements and decisions. While expertise in such fields as medicine, 
physics, and chess have been studied intensely during the past twenty years (e.g., Kleinmutz [1968], Einhorn [1970], Elstein et al. [1978], Johnson et al. [1982]), comparatively little research has been done in the fields of business and management. Particularly in the field of auditing there is a lot of interest in how auditors make judgements.

Expertise has been defined as the "knowledge about a particular domain, understanding of domain problems, and skill about solving some of these problems" [Hayes-Roth et al., 1983]. Davis has proposed that the nature of expertise includes the ability to: (1) solve the problem; (2) explain the result; (3) learn; (4) restructure knowledge; (5) break rules; (6) determine relevance; and (7) degrade gracefully [Davis, 1982].

An expert's knowledge consists of both public and private information. Public knowledge includes the facts, theories and definitions as found in the texts and journals referenced by those studying in the domain. However, human experts also possess private information that is not found in any of the public literature. Much of this private knowledge is in the form of rules of thumb which we will refer to as heuristics. Heuristics allow experts to "make educated guesses when necessary, to recognize promising approaches to problems, and to deal effectively with errorful or incomplete data" [Hayes-Roth, 1983]. Knowledge engineers, who are concerned with the acquisition and representation of knowledge, concentrate much of their effort on the elucidation and reproduction of such "rules of expertise." Human expertise in problem solving is largely the recognition and use of heuristics. Feigenbaum emphasizes that "experience has taught us that much of this knowledge is private to the expert, not because he is unwilling to share publicly how he performs, but because he is unable. He knows more than he is aware of knowing" [Feigenbaum, 1978].

Experts hypothesize possible solutions early in the problem solving process whenever possible. In medical diagnoses, for example, it has been shown that many errors are the result of a failure to include the correct diagnoses in the early hypotheses considered. The medical profession has commonly encnuraged med- 
ical workup sheets, which serve to increase the number of hypotheses considered and help to avoid premature closure. Accounting firms have similarly used generalized control questionnaires and standard audit planning worksheets to help auditors analyze various types of exposures that may occur.

Auditors may be considered experts in performing certain tasks. The objective of this study is to determine the processes that auditors use in a specific audit task, formalize and implement those processes as a computational model, and then test the model.

\subsection{Auditing Internal Accounting Controls}

The American Institute of Certified Public Accountants (1979) defines internal controls as "the plan of organization and all the coordinate methods and measures adopted within a business to safeguard its assets, check the accuracy and reliability of its accounting data, promote operational efficiency, and encourage adherence to prescribed managerial policies..." (Section 320.09) [AICPA, 1972]. Mair, Wood, and Davis in their book "Computer Control and Audit" present the definition: "Controls act upon things that can go wrong which, in turn, leads to the reduction of exposure" [Mair et al., 1978]. Although all public accounting firms evaluate controls and general guidelines have been suggested by several different researchers [Mautz \& Winjum, 1981], auditors still have difficulty evaluating the quality of internal control systems. Because the extent of audit work to be performed by external auditors is determined in large part by an evaluation of internal controls, there is a need for a more rigorous framework of internal control evaluation. Further, the Foreign Corrupt Practices Act (1977) places particular emphasis on the existence of, and the auditor's evaluation of internal controls.

The evaluation of internal controls has typically used such decision support aids as flowcharts, questionnaires, and narratives concerning the client's accounting systems. After studying the flowcharts and questionnaires, the auditor makes a judgement as to the relative strengths and weaknesses of the internal control system. The judgement process of evaluating problems in the 
system of internal controls from flowcharts and narratives is still a "subjective art" at best. Although experts can provide a novice with some logically sound methods of processing the available data, they cannot tell the novice how to evaluate the strength of an internal control system. In fact, experts themselves sometimes do not agree on what constitutes adequate control. Recently, much research has focused on judgemental consensus among auditors [Joyce and Libby, 1982 and Libby, 1981].

The weaknesses inherent in these traditional techniques have been recognized for some time by accountants and accounting firms. The last several years have witnessed the introduction of a number of new approaches intended to regularize the data collection and evaluation process. Previously referenced research suggests a greater likelihood of success on the collection side than on the evaluation side. Nevertheless, public accounting firms hope that these new approaches will lead to greater consensus in evaluating internal controls among their field auditors.

Libby has suggested that "fault trees could be constructed to indicate important potential errors and the controls designed to detect them. These trees would highlight the key controls and also focus on their interrelationships" [Libby, 1981]. The concept of a fault tree is similar to, but a naive version of, hierarchically ordered production-rules as used in current expert systems.

4.1.1. The Audit Task

The study and evaluation of internal accounting controls is a problem involving the expertise of well-trained auditors. The task of determining the presence (or absence) of accounting controls is a requirement of each and every audit performed by CPAs. The following discussion elaborates on the auditor's task in auditing internal controls. The discussions also attempt to sketch out auditor strategies in order to suggest the means by which a model of the auditor's expertise might be constructed.

The general objective in studying accounting internal controls is to satisfy the auditor's second standard of field work: "There is to be a proper study and evaluation of the existing internal 
control as a basis for reliance thereon and for the determination of the resultant extent of the tests to which auditing procedures are to be restricted" [AICPA, 1972]. Thus the PRIMARY purpose is to determine whether the accounting controls are strong enough to be relied upon to produce reliable financial information. If the internal controls are determined to be strong, then the scope of other audit procedures may be more restricted than when the internal controls are determined to be weak. A second objective is to provide the auditor with a basis for constructive suggestions on how to improve the client's internal accounting controls [Loebbecke and Zuber, 1980].

\subsubsection{Task Expertise}

The strengths and weaknesses of an internal accounting control system are evaluated by determining control objectives, identifying controls and faults from a description of the system, and then combining the controls and faults into an overall evaluation of the sufficiency with which each control objective has been met.

Controls and faults are conceptual objects that can be identified by particular recognizable patterns of data embedded within the statements describing the accounting information system. Controls are patterns of data that prevent, correct, or detect system exposures to loss or misrepresentation. These patterns may be of various sizes. Faults, however, act as "red flags" triggering auditor concerns. The identification of a fault does not necessarily indicate a system weakness as there may be offsetting controls, but the identification of a fault generally results in a search for compensating controls.

Auditor associations between specific system designs and the resulting strength of internal controls, are learned through formal education, case examples, and by performing many similar auditing tasks. These associations permit chains of deductive reasoning to be constructed connecting the structural design of the accounting system and the likely functional strengths and weaknesses of the system. This approach is called prototypic reasoning. Based on the pervasiveness of prototypic reasoning among experts and the manner in which auditing expertise is 
acquired, we will proceed on the assumption that auditors use prototypic reasoning [see Figures 6 through 13].

The result of the internal control evaluation task consists of: (1) a suggested list of controls for the compliance testing phase; and (2) a list of control weaknesses. The list of control weaknesses indicates significant problems discovered during the evaluation process and the resulting exposures that could occur. The auditor uses this list in establishing subsequent compensating audit steps and for interaction with management. The specific weaknesses identified are combined with the controls to determine sufficiency for each control objective, which in turn results in the auditor expanding some of the substantive tests performed later in the audit.

\subsubsection{Task Difficulty}

The overall system evaluation of internal accounting controls is difficult. There is no unique set of acceptable controls that is considered normative. Rather, the accounting system may be configured with numerous acceptable combinations of controls. Also, experts are unable to describe each step in the evaluation process, rather the process is normally taught by having students solve numerous case problems and provide ex post rationalizations of the analysis process followed. Behavioral studies clearly indicate that the ex post explanation often does not match the process actually followed by the expert [Nesbett and Wilson, 1977].

\subsection{The Research Project}

The objective of this study is to formulate and test a model of the processes employed by audit managers and partners in reviewing and evaluating internal accounting controls. Development of the model will be based on: (1) interviews with a small sample of practicing auditors; and (2) observations of these same auditors performing the internal control evaluation review task. The resulting model will be implemented as a computational model and tuned to one expert. The model output will consist of two lists: (1) recommendations for specific controls to be compliance tested; and (2) a list of control weaknesses. The model will then 
CONCEPTUALIZATION

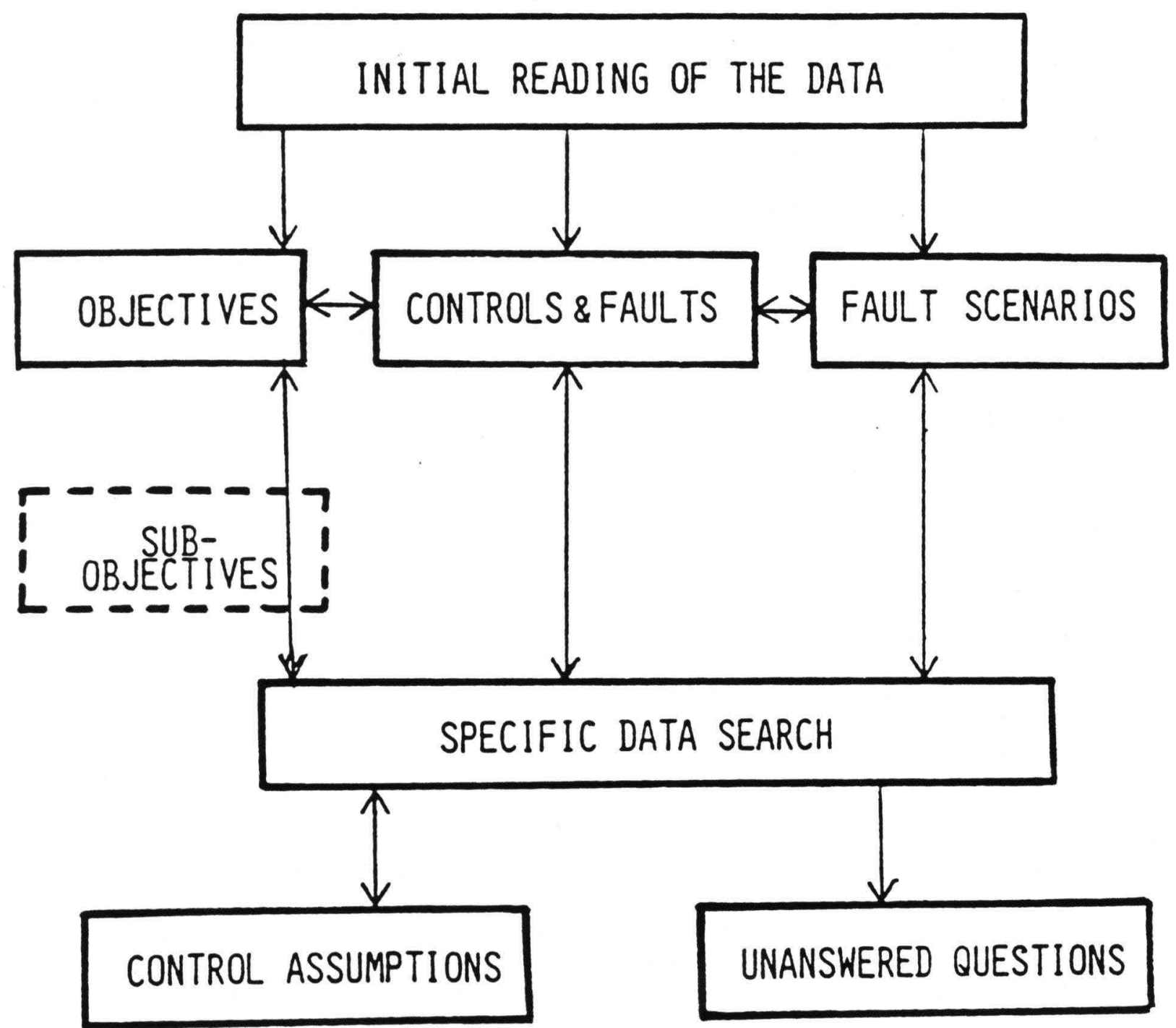

Figure 6 
SCENAR IOS

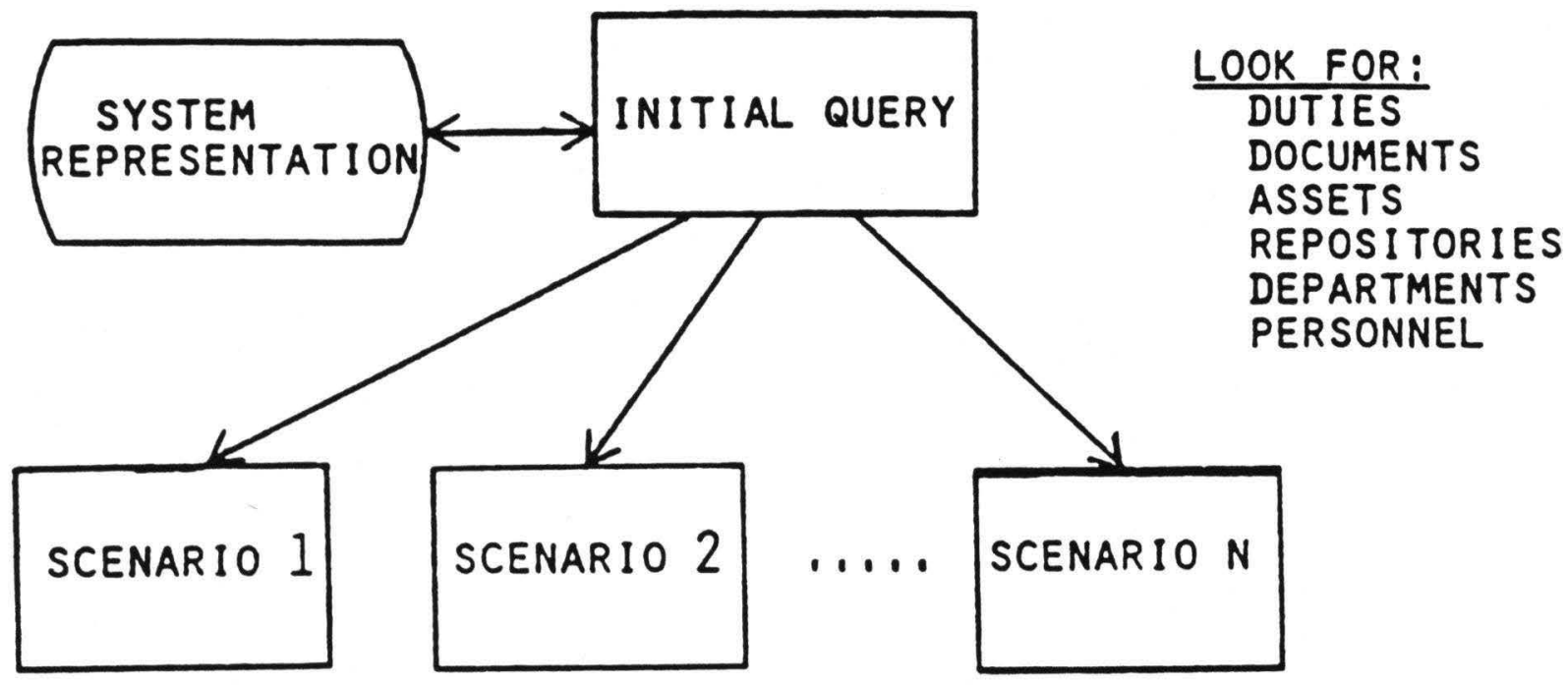

A SCENARIO IS

A HIGH LEVEL EXPECTATION OF WHAT DOES/MAY

HAPPEN WITHIN SOME ACCOUNTING ROUTINE.

THIS SUGGESTS LIKELY

1. EXPOSURES

2. CONTROL OBJECTIVES

Figure 7 
CONTROL OBJECTIVES

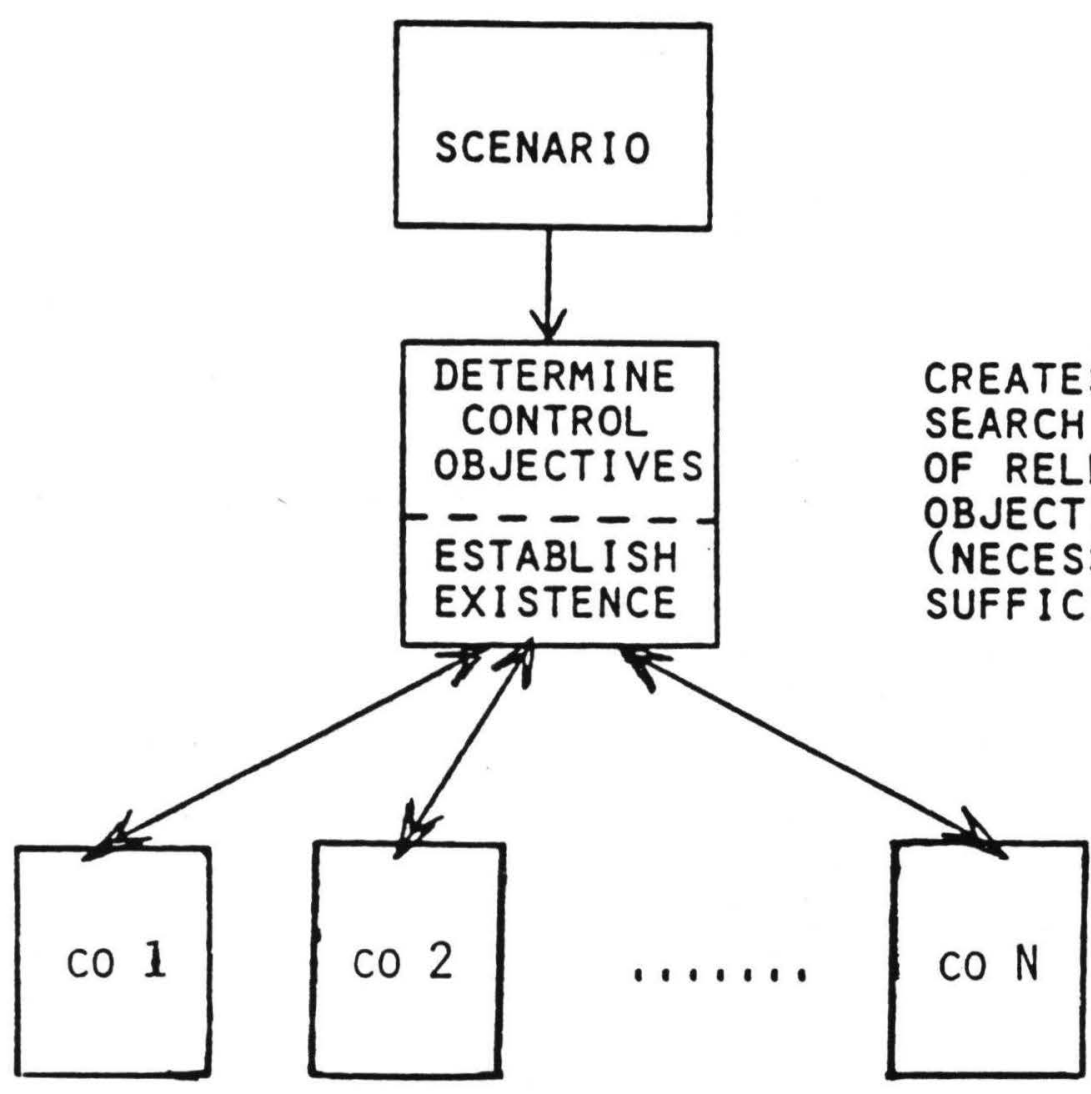

A CONTROL OBJECTIVE IS

A HIGH LEVEL ABSTRACTION OF WHAT PROTECTIVE/DETECTIVE DEVICES SHOULD BE USED.

CONTROL OBJECTIVES MAY BE BROKEN INTO CONTROL OBJECTIVE SUBGOALS. 
CONTROL PROCEDURES

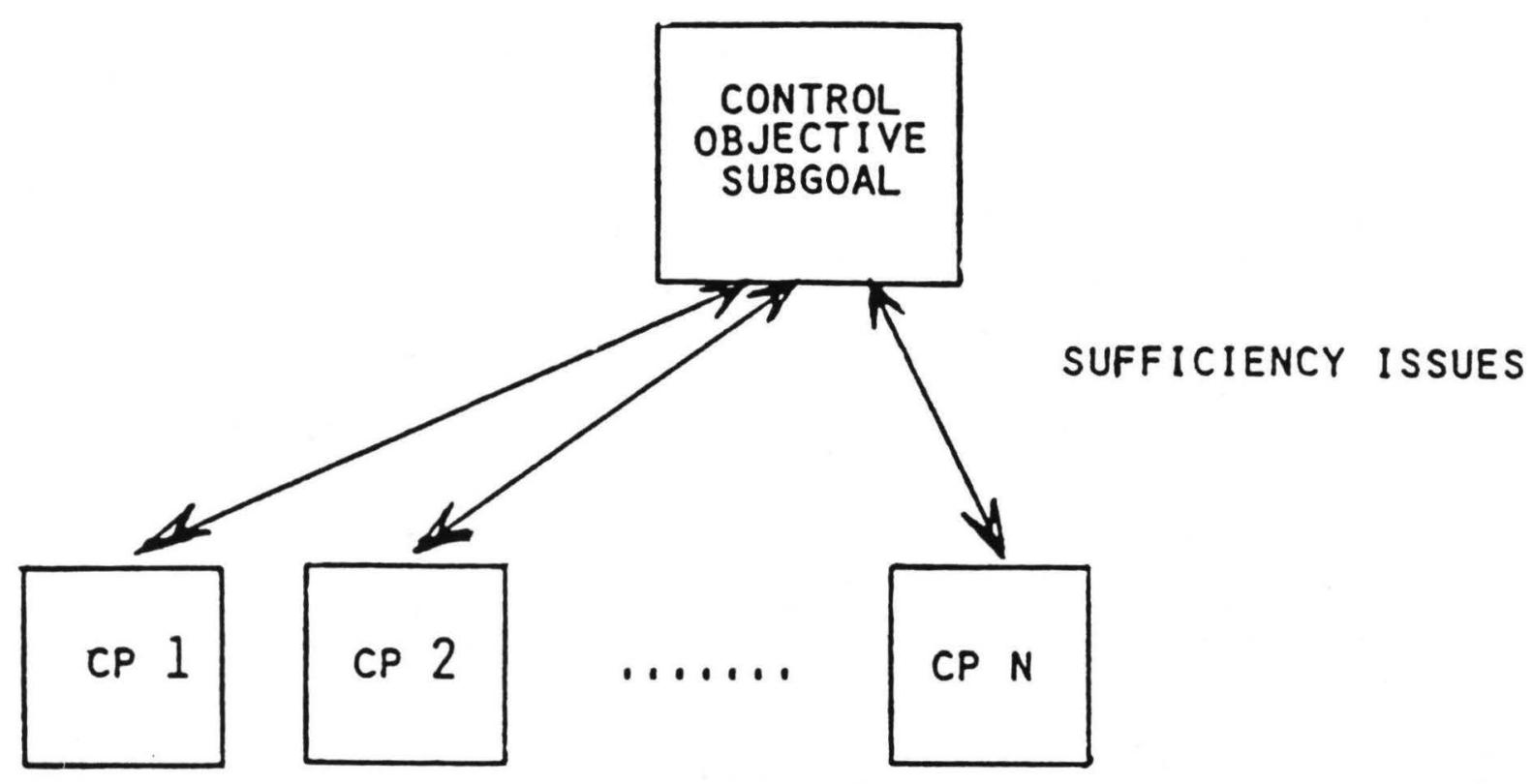

CONTROL PROCEDURES

1. LOOK FOR AN IMPLEMENTATION (A MATCHING PATTERN)

2. ARE THE LOWEST LEVEL OF CONTROL KNOWLEDGE.

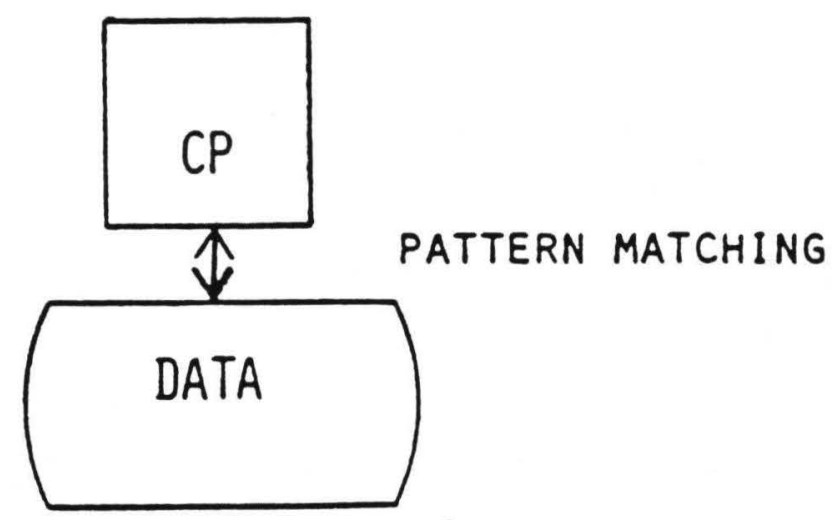

Figure 9 


\section{CONTROL EXAMPLE}

CONTROL OBJECTIVE: CONTROL SHOULD BE ESTABLISHED OVER GOODS AND SERVICES AS A BASIS FOR:

1. DETERMINING AND RECORDING LIABILITY FOR GOODS AND SERVICES RECEIVED BUT NOT RECORDED. 2. POSTING ITEM TO DETAILED INVENTORY RECORDS.

CONTROL OBJECTIVE SUBGOAL: RECEIVING REPORTS SHOULD BE CONTROLLED IN SUCH A WAY THAT IT CAN BE ESTABLISHED that all Related transactions have been accounted FOR WITH RESPECT 10 GOODS.

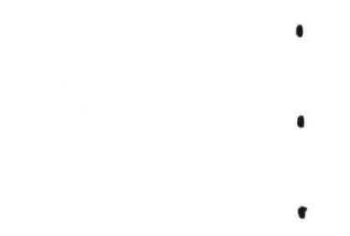

CONTROL PROCEDURE: [ESTABLISH THAT ALL RECEIVING REPORTS HAVE BEEN ACCOUNTED FOR.] 
CONCEPTUALIZATION OVERVIEW

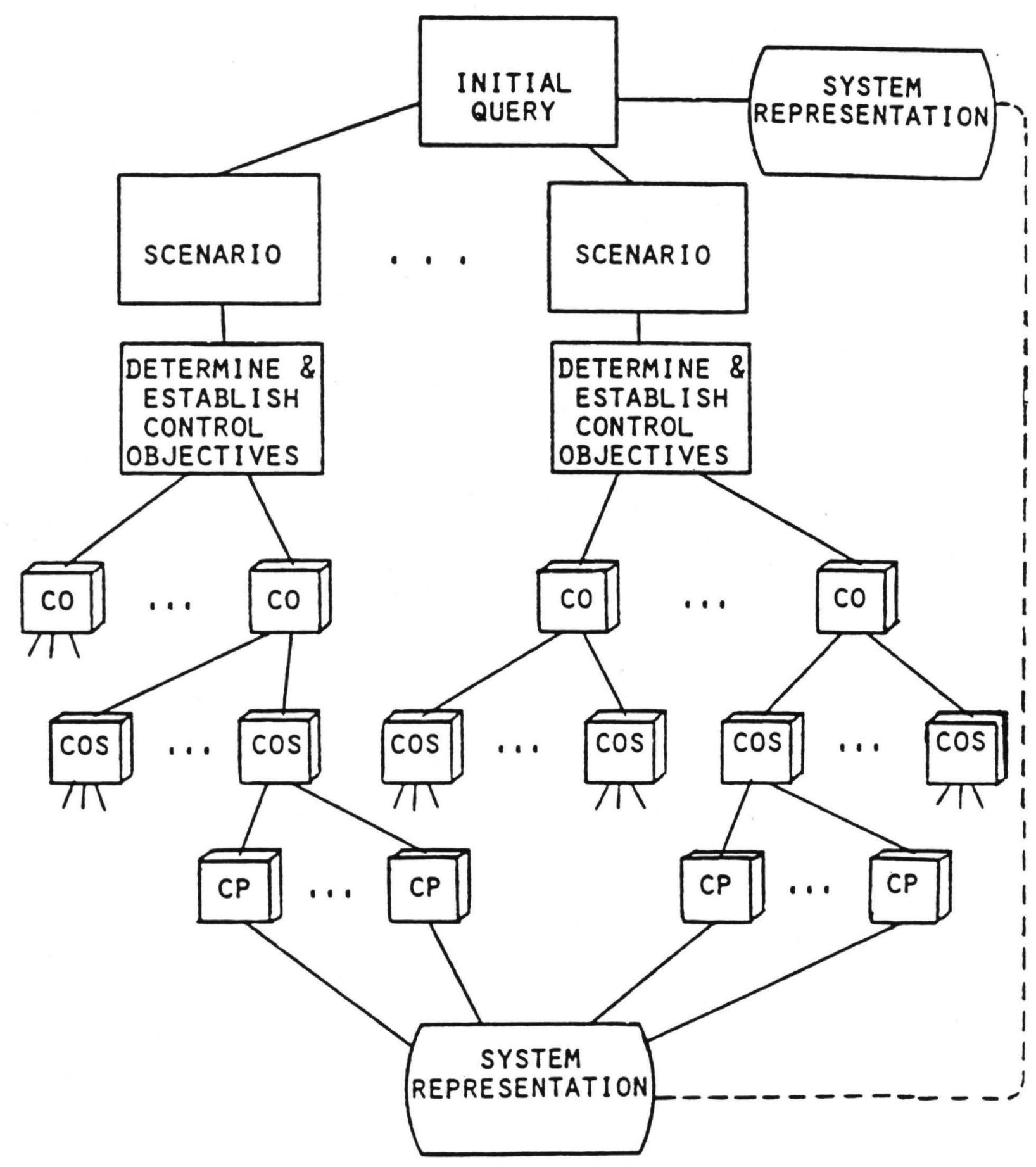

- not necessarily a tree (overlap)

Figure 11 


\section{STRUCTURE}

OBJECTS: REPRESENT FACTS AND CONJECTURES ABOUT THE PROBLEM

PATTERNS: OBJECTS THAT DESCRIBE FEATURES OF OTHER OBJECTS (CONTROLS \& FAULTS)

HYPOTHESES: OBJECTS THAT REPRESENT HYPOTHETICAL PROTOTYPE SITUATIONS (OBJECTIVES, SUbobJeCtIVES, \& FAULT SCEnARIOS)

ACTIONS: EXECUTABLE PROCEDURES THAT SPECIFY CHANGES TO THE HYPOTHESES

RULES: ASSOCIATIONS BETWEEN PATTERNS AND ACTIONS

Figure 12 


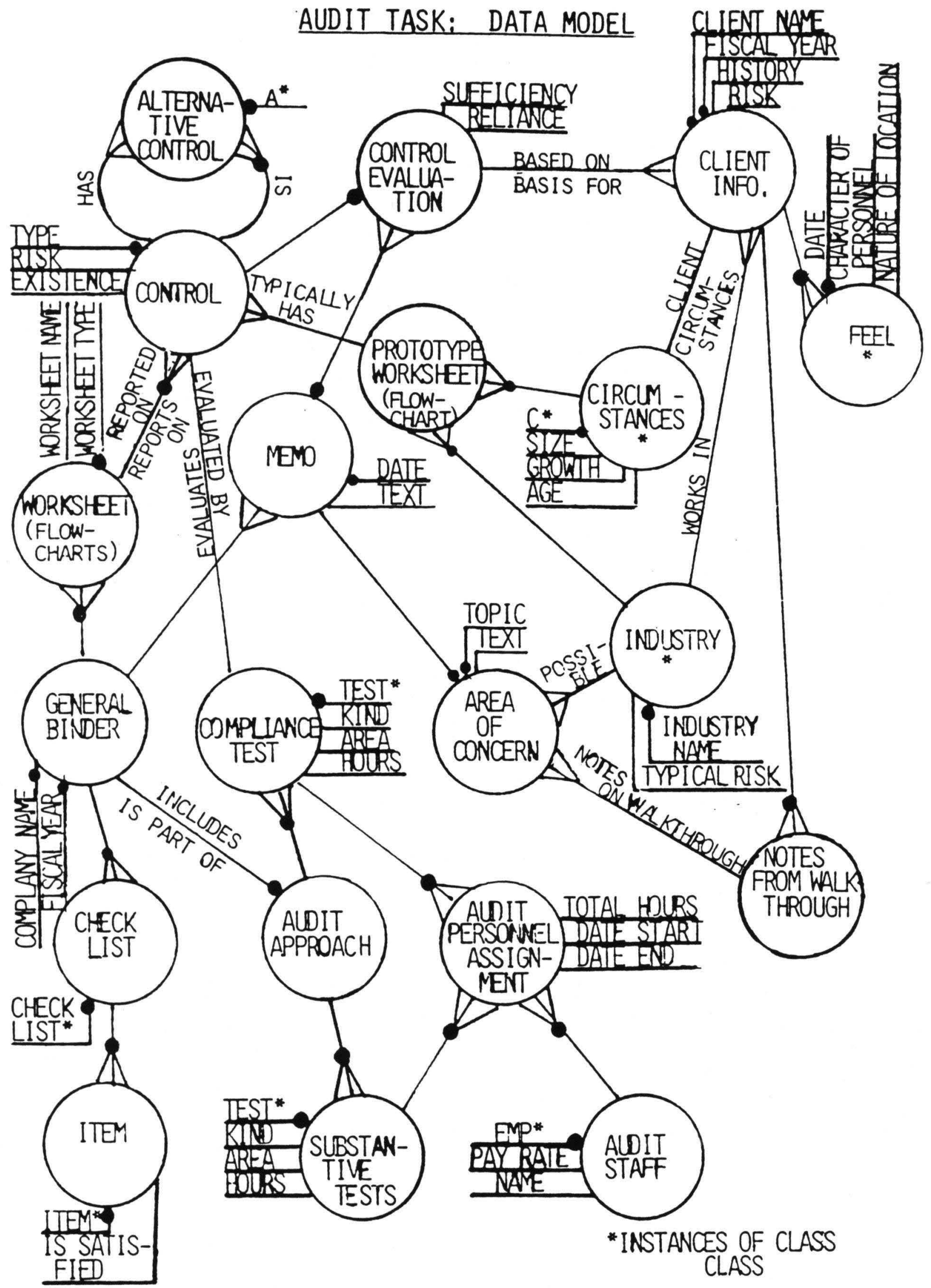

Figure 13 
be tested using new cases (same task) and cross-validated against the performance of additional auditors performing the task.

\subsubsection{Limitations}

The initial model will be limited to evaluating controls commonly found in Purchases, Payables and Cash Disbursement systems. This is consistent with generally accepted auditing standards (Section 320), which emphasizes an ordered approach to considering classes of transactions as follows:

A. Sales, Receivables and Cash Receipts;

B. Purchases, Payables and Cash Disbursements;

C. Inventories and Production;

D. Personnel and Payroll;

E. Property, Plant and Equipment.

In addition, the initial computational model will not be expected to handle novel (uncommonly different) accounting systems.

\subsection{Project Phases}

The objectives for the research program are summarized in three phases: (1) model development, (2) model implementation and tuning, and (3) model evaluation. (See Figure 14 with discussion below.)

4.3.1. Phase I-Model Development

Preliminary representations of expertise, including key concepts and relationships, have been developed from interviews and experimental task data using experts both as collaborators and as subject-informants. These descriptions consist of problem solving steps and heuristics that represent auditor judgement in (a) identifying internal accounting control objectives, (b) identifying controls and faults in the accounting system, and (c) evaluating the system controls, weaknesses, and sufficiency of documentation.

4.3.1.1. Knowledge acquisition

Research at the University of Minnesota by Johnson [1983] has isolated general principles of eliciting expert knowledge that 
$P L A$ II OF HOPK

$\| O D E L$ DEVELOP\|ENT PHASE

PROTOCOL SCORING RULES

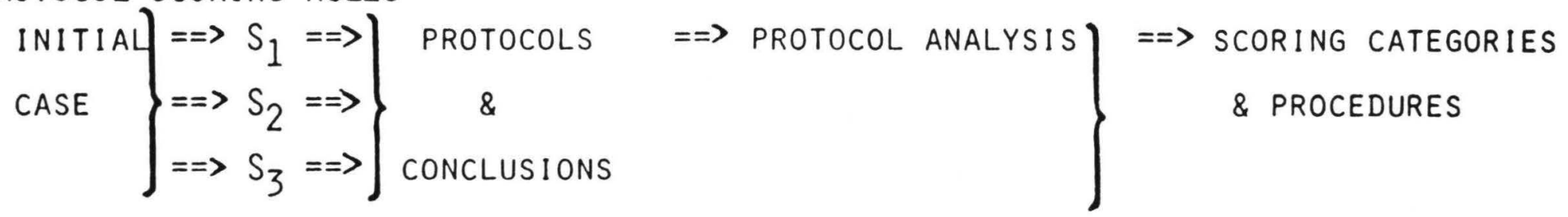

MODEL DEVELOPMENT

$$
\begin{aligned}
& \text { ADDITIONAL CASES } \Rightarrow S_{1}=\Rightarrow \text { PROTOCOLS \& } \Rightarrow \text { SCORED PROTOCOLS } \Rightarrow \text { HEURISTICS } \\
& \text { (OBSERVATIONAL) CONCLUSIONS \& CONCLUSIONS (KNOWLEDGE STATES } \\
& \begin{array}{ll}
\text { INTERVIEWS } \quad \Rightarrow S_{1} \Rightarrow \text { HEURISTICS } \quad \Rightarrow \text { PROCESSES) } \\
\text { (DESCRIPTIVE) }
\end{array} \\
& \text { (KNOWLEDGE STATES \& PROCESSES) } \Rightarrow \text { FORMALIZED AS THE } \\
& \text { MODEL }
\end{aligned}
$$

MODEL TUNING PHASE

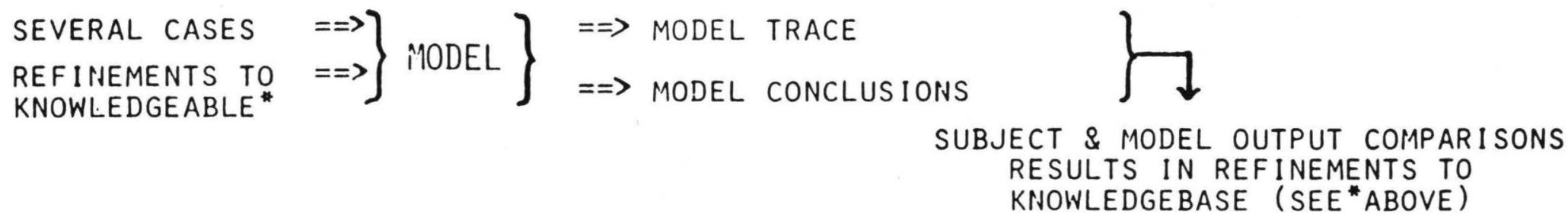
SEVERAL CASES $==>S_{1}=>\begin{gathered}\text { PROTOCOL } \&==>\text { SCORED PROTOCOL } \\ \text { CONCLUSIONS }\end{gathered}$

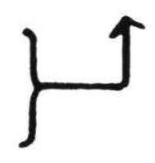

Figure 14A 
PLAN OF WORK (CONTINUED)

MODEL VERIFICATION PHASE

GENERAL DATA

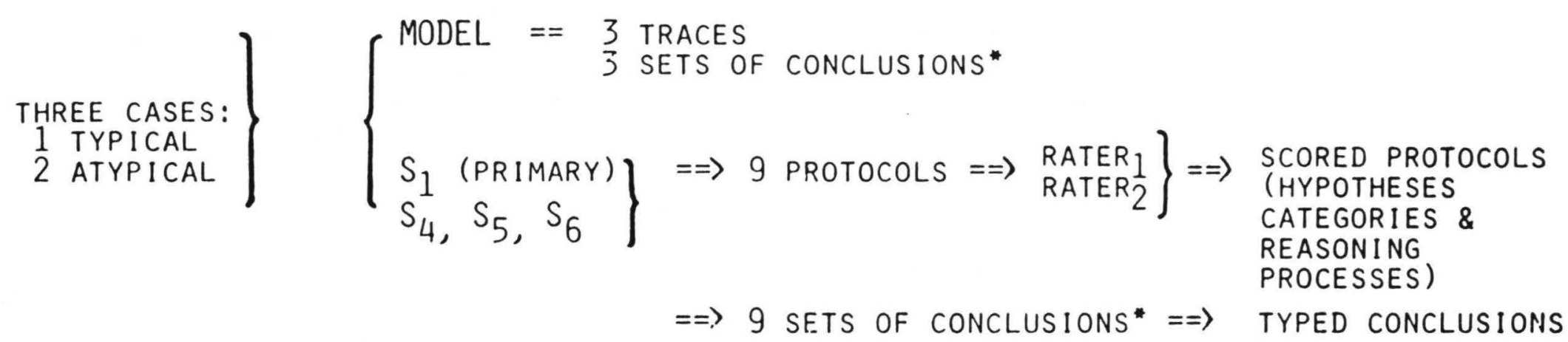

PEER JUDGMENT

$$
\begin{aligned}
& 3 \text { MODEL TRACES } \\
& 3 \text { SETS OF MODEL CONCLUSIONS* } \\
& \begin{array}{l}
12 \text { SCORED SUBJECT PROTOCOLS } \\
12 \text { SETS OF SUBJECT CONCLUSIONS (TYPED)* }
\end{array}
\end{aligned}
$$

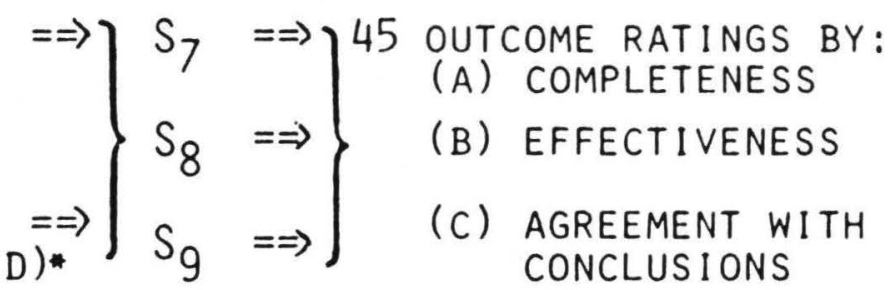

"CONCLUSIONS CONSIST OF:

(1) LISTS OF CONTROLS TO BE COMPLIANCE TESTED

(2) LISTS OF CONTROL WEAKNESSES 
may apply in a variety of settings. This research continues to develop and use these approaches to elicit knowledge from our expert auditors. The multi-method approach is summarized here in three parts.

4.3.1.2. Observational methods

The observational approach adopted for this study is to collect "thinking-aloud protocols" which attempt to probe the problem solving mechanisms being used by experts. These protocols provide information about the organization of the expert's knowledge base, the actual knowledge it contains, and what control structures are used to apply the knowledge. A major difficulty with observational methods is that the very techniques used to determine the reasoning processes may distort those processes [Nesbett and Wilson, 1977]. That is, the line of reasoning chosen by the auditor when asked to relate each thought in the process while solving the problem may be different from the one used when that same auditor solves the problem under more natural conditions [Johnson, 1983a]. In this study, subjects are asked to solve several cases using "thinking-aloud protocols." The protocols are then transcribed and analyzed to determine what processes are being used. The resulting data is formalized as a set of rules. This initial protocol phase of research is followed by a refinement phase in, which experts are asked to comment on the preliminary models developed from the protocols.

\subsubsection{Descriptive methods}

Using this method, assessing expertise is essentially a formalization process in which portions of the knowledge of an expert are transformed into an explicit representation. One method of accomplishing this is through interviews with auditors in which they attempt to characterize their knowledge and skill in the given task situation. The type of questions that are asked include the following:

1. "What objectives $h$ do you think of when you see problem data about $c$ ?"

2. "What other evidence makes you start thinking about ob- 
jective h?"

3. "What do you do immediately after thinking about objective h?"

4. "What evidence (controls or sub-objectives) makes you more certain that objective $h$ is satisfied?"

5. "What do you do when you see evidence that supports objective h?"

6. "What evidence makes you conclude that objective $\mathrm{h}$ is satisfied?"

7. "What do you do once you know that objective $h$ is satisfied?"

8. "What evidence makes you less certain that objective $h$ is satisfied?"

9. "What do you do when you see faults in the data that contradict objective h?" (What compensating controls do you look for?)

10. "What makes you conclude that objective h cannot be satisfied?"

11. "What do you do once you know that objective $h$ cannot be satisfied?"

12. "What evidence makes you conclude that you have sufficiently solved the problem?"

13. "What evidence makes you conclude that you cannot solve the problem?"

14. "What do you do when you've seen all the problem data and are still unable to solve the problem?" [Moen, 1984, p. 51].

A second, more formalized means is through the creation of a precise "language" in which the expert describes his/her expertise. The structure of the language is determined by the architecture of the knowledge base. In this case an auditor describes his/her lines of reasoning by means of production rules, if-then statements. Most existing methods of assessing expertise rely heavily on descriptive methods. The major limitation with the descriptive method is that the more competent an expert becomes, the less able he is to describe his problem solving knowledge [Johnson, 1983]. Several books have been written on the objectives, rules and procedures for evaluating internal controls [Johnson and Jaenicke, 1980]. Using descriptive methods, expertise 
has previously been captured in the questionnaires and workpapers of the large accounting, firms. Although these books provide a reference for basic internal control production rules, this study will emphasize those rules elicited directly from the experts rather than from the literature. To the extent rules identified in the literature can be confirmed by auditor performance, such rules may be used.

\subsubsection{Intuitive methods}

Intuitive methods for capturing knowledge exist in two forms. In one case, a knowledge researcher interacts with both the auditor and the literature of the field in order to become familiar with its major problem solving methods. Acting in this capacity, the researcher develops a representation of auditing expertise which is then checked against the opinion of other auditors and eventually incorporated into our computer program. A second intuitive method of knowledge capturing is where a researcher, who is an expert in the area, attempts to describe the basis for his own knowledge and skill. Intuitive methods are less constrained by notation structures than the descriptive analysis techniques which utilize separate auditors and researchers. However, they still suffer from the fact that dependence on intuition as a means of recovering one's own knowledge is subjective and may not be adequate [Johnson, 1983]. Intuitive methods will be used in the model building process to supplement the above described observational and descriptive methods when necessary.

\subsubsection{Subjects}

Practicing CPAs in middle-managment of a large auditing firm are serving as subjects in the project. The local office of an international auditing firm has provided time from busy manager and partner schedules to participate in both the model building and the cross-validation. The model is being built largely with the help of one auditor, who will also assist in model validation. In addition, six other auditors will assist in validating the model. 


\subsubsection{Knowledge acquisition summary}

The study began with the creation of a "zero-order" model of auditor processes. The model is then being developed as follows:

1. Interviews (descriptive methods) are being conducted with subjects to gather information and resources for the development of specific task materials and to develop preliminary descriptions of expertise. Experts are used as both collaborators and subject-informants.

2. Experimental tasks are employed to assess expertise (observational methods). Subjects in these tasks attempt to generate relevant internal accounting control objectives and evaluate working papers while "thinking aloud." Transcripts are made of problem solving sessions and these are being analyzed in detail following procedures developed by Erickson and Simon [1984], Johnson at Minnesota [Johnson, 1983], and refined as part of the research.

3. A model of auditor expertise is then being formalized as a set of rules (see the knowledge representation discussion below) and further refined using descriptive methods.

The products of the knowledge acquisition portion of the research includes the representations of auditor expertise in (a) the identification of specific internal accounting control objectives; (b) the evaluation and review processes, identifying which controls should be further tested for reliance thereon; and (c) the type of processes used in recognizing controls and weaknesses.

\subsubsection{Knowledge representation}

We have chosen to represent the expert knowledge of an auditor by the use of production rules. We make no claim for the universal applicability of such a representation, but rather rely on the demonstrated utility of rule-based systems with characteristics similar to those encountered in analyzing internal controls. Rule-based representations (also referred to as situation --> action rules or IF --> THEN rules) allow easy modification and explanation, both considered essential for building and then 
tuning such computational models. Basically, each rule must capture a "chunk" of the domain knowledge, meaningful in and of itself to the domain specialist [Feigenbaum, 1978]. The triggering conditions are normally referred to as the antecedents. The context is the "scratchpad" or working space describing the current state of the problem being solved. If the antecedents are matched against the context, then the consequences are enacted.

The rules are normally associated with "lines of reasoning" and "episodes" that are comprehensible to the domain expert [Feigenbaum, 1978]. Lines of reasoning involve the system analysis methodologies emoloyed by the subject. Episodes involve proposing tentative goals and/or subgoals (hypotheses) and trying to either substantiate or disprove the goal. Such a generate-andtest framework has been identified in behavioral studies by Biggs and Mock [1981] and others when studying audit settings. The formulation and maintenance of lines of reasoning and episodes often requires the integration of many different "chunks" of knowledge. It is important that the implemented system be able to explain its use of knowledge to the domain expert for both refinement and validation purposes.

The knowledge representation portion of the model development involves encoding the specific expertise identified (part one) into production rules along with additional domain knowledge. Each production rule represents a meaningful "chunk" of either specific expertise and/or of general domain knowledge.

\subsubsection{Phase II-Model Implementation and Tuning}

The preliminary model developed in Phase I is being implemented as a computational model by adapting a modelling tool, Galen, developed at the University of Minnesota [Thompson et al., 1983]. Galen's architecture reflects its development in modelling cognitive processes. Galen's inference engine has the ability to partition the knowledge base, the ability to search for a hierarchical set of goals, apply forward and backward chaining, and can interact with a LISP representation of the audit working papers. While demonstrating the generality of Galen, we find that the adaptation of an already proven tool enhances our productivity. 
Combined with the rules developed in Phase I, the product will be the shell of our computational model.

After the knowledge from Phase I is mapped into GALEN's representational framework, the system will be "tuned." Tuning involves running several prototype accounting information systems through the evaluation process and, in collaboration with our expert, checking the lines of reasoning and episodes for reasonableness and making adjustments in the rules.

\subsubsection{Phase III-Model Evaluation}

Phase III experiments will focus upon comparisons between the control evaluation strategies of the model and the processes employed by auditors. The framework we propose to use to evaluate the model performance has two major features: (1) tests of sufficiency or adequacy of model outcomes; and (2) tests of the quality of model processes and of cue usage. [See Figure 14]

Researchers have not been able to formulate any single critical experiment to which validation of similar models are susceptible. Furthermore, due to the small sample size, statistical evaluations of experimental results are generally not available and researchers are constrained to rely on graphical techniques. The approach used in this study will include several empirical tests. The empirical tests address different types of data, each of which speaks to a different aspect of the model behavior.

The computational model will be fine-tuned around the expertise of one individual auditor (the primary subject). The computational model will initially be validated against this individual using three cases which were not part of the model development phase. In addition, the model will be cross-validated against three additional auditors using the three new cases.

For each case, the primary subject and three other auditors will be asked to read aloud the pertinent data and give "thinkingaloud" protocols while reviewing and evaluating the workpapers prepared by an in-charge auditor. As part of the task, the subjects will be asked to write out a list of: (1) recommendations for specific controls to be compliance tested; and (2) weaknesses 
identified from the system description. At the conclusion of each case, subjects will be asked to fill out a participant background questionnaire. The complete problem solving session for each subject will be tape recorded and transcribed. The computational model will also receive each case and make similar evaluations. The output of the model will include: (a) recommendations for specific controls to be compliance tested; (b) weaknesses identified from the system description; and (c) a trace of all data analyzed and rules fired.

4.3.3.1. Tests of adequacy of model outcomes

The first type of analysis, for sufficiency or adequacy of model outcomes, is to establish that the computational model can identify and evaluate internal accounting controls. Outcomes to be examined include the lists described above. The model's and all four subjects' outcomes will be retyped and given to another three subjects to judge. For each task, the three subjects will rate the (a) model's and four subjects' evaluations lists, (b) subjects' scored protocols, and (c) model's trace. These outcomes will each be rated on three seven point scales: (1) completeness of review; (2) effectiveness of compliance tests selected; and (3) agreement with review conclusions. These ratings will then be analyzed to determine how well the model performs in relation to the person it is modelled after and in relation to the other auditors.

4.3.3.2. Tests of quality of model processes and cue usage A second type of analysis involves establishing the quality of the evaluation processes employed by the model and the usage of critical cues. To establish quality of evaluation processes, the inferences made must not only be "legal", but be the type of inferences that experts would make. Determining that a sample of model behavior constitutes adequate auditing behavior is not a simple matter. In games, such as chess, it is fairly easy to determine if the model is performing the requisite behavior because the rules used to determine whether a given move is "legal" are well-defined. By contrast, in environments such as 
fault diagnosis and internal control evaluation, it is not clear what constitutes a "legal move." Furthermore, as in a game, though all reasoning steps are explainable by logic or rules, some "lines of reasoning" (smaller sets of steps or moves) must be made according to a criterion of quality in order for the task to be well done. In medical diagnosis, for example, there is typically present a small set of cues that if interpreted properly will lead to a correct diagnosis. Experts may differ in their interpretations of other pieces of information, but they tend to agree more on the interpretation of these critical cues and use additional cues to mitigate between competing hypotheses [Johnson et al., 1982].

The model evaluation framework adopted requires that the model's rules for performing the evaluation be found in the heuristics of expert behavior. The judgement as to whether the model is performing the task is then based upon a comparison between the specific acts of model behavior on the task and by the behavior of the expert auditors. The comparisons focus upon ( 1 ) the identification and use of specific goals and objectives which direct the search and confirmation processes, and (2) knowledge states, and the cognitive processes that link them. The model quality will be evaluated by transcribing, scoring, and analyzing each model trace and verbal protocol selected. Protocols provide a depth of understanding about judgement and decision making unavailable using other methods. However, as in other methods, the data must be reduced to a structured, objective image of the processes that auditors are using. The analysis is developed by synthesizing the results of two analytical methods: a topdown, global analysis and a bottom-up, knowledge state/cognitive process analysis.

The top-down analysis identifies single problem solving goals from the protocols. Proposed categories are developed through functional analysis of the review task, formalized descriptions of evaluation processes generally, and model fragments found in the auditing literature. An example may be the representation or mental picture of the segregation of duties within the purchasing/ cash distribution function. The identification of problem solving 
goals establishes the boundaries within which the more detailed state and process analysis is performed [Melone, 1984].

The bottom-up analysis focuses on knowledge states, the basic set of facts, concepts and hypotheses generated by the subject, and an associated set of reasoning processes called cognitive processes (e.g., reading, requesting information, searching for information). Scoring protocol for reasoning processes permits an understanding of how auditors use old knowledge states, generate new knowledge states, and the type of processes that link the knowledge states together. This analysis provides a "picture" of the path taken by the auditor through his representation of the task. It differs from the top-down analysis primarily by being more elementary and in finer detail [Melone, 1984]. An example of an operator would be the "comparing" of two items. By associating knowledge states and reasoning processes, a "problem behavior graph" will be developed [Newell and Simon, 1972].

A third, more general type of analysis, "lines of reasoning", will then be determined by analyzing the "problem behavior graphs" and the sequence of problem solving goals. Lines of reasoning involve the methods employed by the subjects to solve the task. Examples would be systemic search and directed search strategies which have been identified in previous auditing research [Biggs and Mock, 1981].

\subsubsection{Validity and consistency}

The transcribed protocols and trace will be scored by two independent raters, trained in the rules for coding these protocols. The coded protocols from each coder will be compared, and the proportion of agreement between the lists developed by each rater for two protocols rated will be computed. Cohen's K [Coken, 1960], an inter-rater reliability coefficient, will be employed to adjust for agreement due to chance. Traces generated by the computational model will be scored by the same methods used to score subject protocols. 


\section{PRELIMINARY RESULTS}

Discussion of subject's decision processes includes several levels of analysis: (1) systemic or directed strategy; (2) episodes; (3) views or frames of reference; and (4) cognitive processes. From previous experience in cognitive modelling, we have found that the decision-making behavior of experts cannot be adequately understood by analyzing cognitive processes alone, rather several higher, controlling levels of analysis are important to the modelling of expertise. The preliminary results from the protocol analysis of our first three subjects can be summarized in the above four categories. The categories range from the more general decision strategy of the subjects, to very specific types of cognitive processes which allow the auditor to progress from one knowledge state to another.

\subsection{Decision Strategy}

Each of the original three subjects used a very systematic breadth-first strategy for their analysis of the working papers and decision making. This has been categorized as a systemic strategy. They first identified all controls and then specified audit procedures and sample sizes. Systemic search contrasts with a more directed or depth-first approach where decisions about controls to test, including sample sizes, are made prior to the complete analysis of the working papers. This finding is consistent with studies by Biggs and Mock [1983] and Biggs, Messier, and Hansen [1985]. In each of those studies, two auditors used the systemic strategy and one auditor used the directed strategy. They observed that in both studies, the "experienced subjects used a systemic strategy" [Biggs et al., 1985].

The finding that expert auditors doing internal control evaluations and reviews use a systemic decision-making strategy rather than a directed strategy may be surprising to some researchers studying expertise. We hypothesize that this may be due to the nature of the particular task. Besides identifying controls, deciding which controls to compliance test, and choosing sample sizes, auditors feel that they examine the entire workpapers in order to catch any serious faults that may exist. 
Also, many of the controls and possible compliance tests are interrelated, requiring an understanding of the complete system and correlation of tests to maximize efficiency and effectiveness. Therefore, a systemic strategy may be the most effective.

\subsection{Episodic Categories}

The next macro level of analysis consists of identifying the larger episodes used by the auditor in the decision-making process. As defined earlier, episodes involve proposing tentative goals and/or subgoals appropriate for the task, and then doing the analysis necessary to either substantiate or disprove the goals. The major episodic categories are presented as a graph in Figure 15. The macro goal categories for the task are: Decide on the likely inherent risk category of client and the most probable overall audit approach to expect. Such firm categorization is based on understanding the macro environment within which the firm operates, firm size, growth, industry, and general management characteristics. (2) Decide if there are significant processing controls which can be relied on and the appropriate compliance tests. (3) Choose which boundary controls for accounts payable to rely on and the appropriate compliance tests. Choose which controls over disbursements can be relied on and the appropriate compliance tests. (5) Evaluate the effectiveness of general computer controls and other firm environment factors when appropriate. (6) Draw conclusions on overall audit approach, controls to be relied upon, and appropriate audit procedures. The above episodic categories are closely related to manner in which the firm-specific workpapers have been organized.

We have chosen to graph these episodic categories by dividing each transcribed auditor protocol into a hundred equal units and then classifying each unit in the appropriate category. As can be seen from the partial protocol graph in Figure 15, the episodes are sustained goal-seeking categories. However, these episodes are somewhat interdependent and must sometimes be suspended until other decisions have been reached. An example is where specific processing controls depend on the computer; before conclusions about the specific tests of computer processing con- 
EPISODES : INHERENT RISK PROCESSING CONTRÖLS......... A/P ADDITIONS. (BOUNDARY). DI SBURSEMENTS . (BOUNDARY)

GENERAL EDP CONTROLS ...... CONCLUSIONS $\ldots \ldots \ldots \ldots$. . . 0
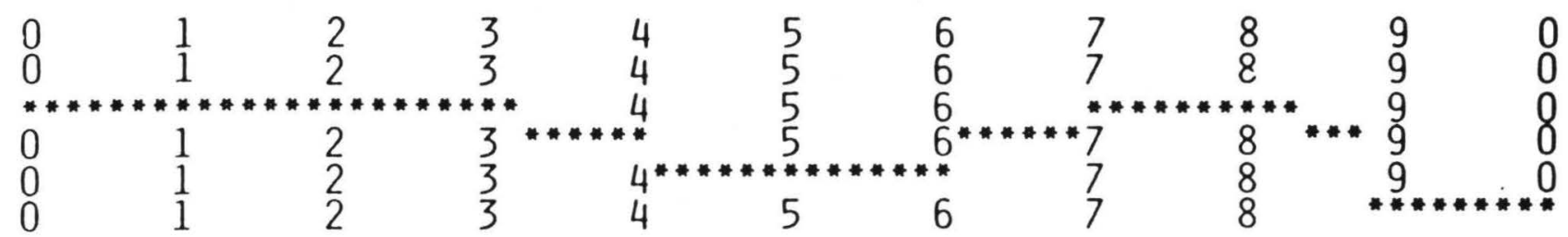

VIEWS OR FRAMES OF REFERENCE

PROCESSING : ........... COMMITMENTS (PO).

RECEIPT OF GOODS \& SERV (RR)O GOODS RET \& CLAIMS ON SUPL 0 A/P ADDITIONS (INV) ...... DI SBURSEMENTS $($ CKS $) \ldots \ldots \ldots$ INVENTORIES . . . . . . . . . . . 0

**

EDP FACTORS ADEQUACY OF WORKING PAPERS

WRITTEN RESULTS:

CONTROLS TO TEST..........

WEAKNESSES AND PROBLEMS ..

\section{CONTROLS:}

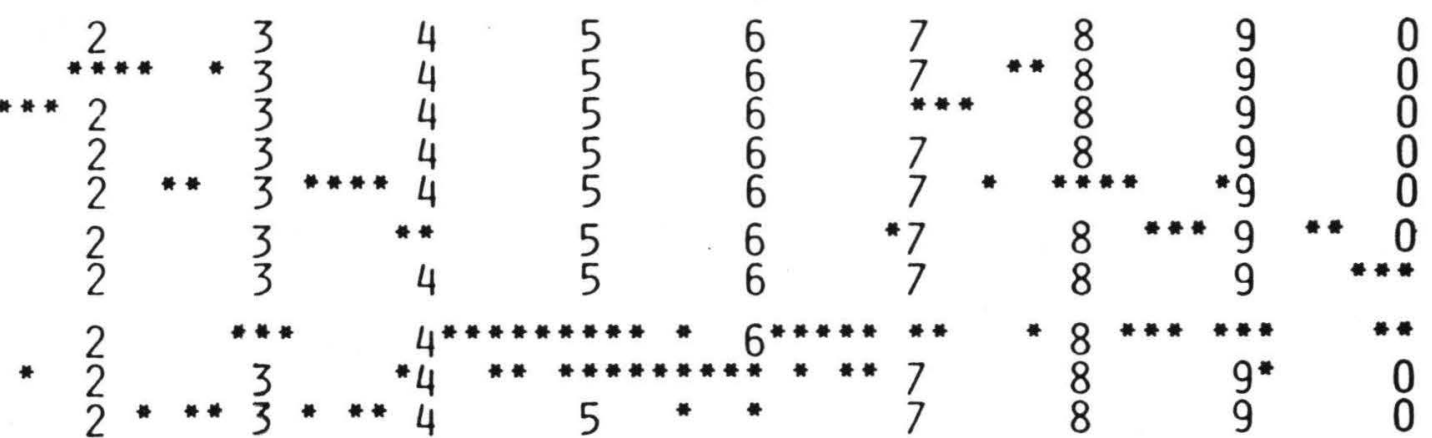

1. PROgRammed COMPARISON OF RECEIVING REPORT WITH POS ON FILE.

2. A/P COMPENSATING PROCEDURE: IF LIMITED SUPPLIERS, CONFIRM A/P AT YEAR END.

3. DO A "PROOF OF CASH" FOR A FEW MONTHS.

WEAKNESSES:

1. POS: NO AUTHORIZATIONS WITH REGARDS TO AMOUNTS, ETC.

2. SEGREGATION-PURCHAS ING AGENT: TOO MANY RESPONSIBILITIES,

3. INADEQUATE CONTROLS OVER BLANK CHECKS.

4. QUESTION: ABILITY TO SET UP A.P FOR GOODS RECEIVED, BUT NO INVOICE RECEIVED.

5. SEgREgATION-A/P CLERK: INITIATES PAYMENT AND RESPONSIBLE FOR RECEIVING GOODS. 
trols can be determined the auditor first determines the effectiveness of the general computer controls and extent that they can be relied upon. The graph allows us to relate how the episodes unfold and their relationship with other activities scored for in the protocols.

\subsection{Views or Frames of Reference}

Auditors appear to have major frames of reference through which they view the data. Such frames of reference allow them to organize and evaluate various aspects of the data cues. The views or frames of reference identified from the auditor protocols are: (1) processing, i.e., the flow of documents and goods through various processing tasks and controls within the firm; (2) segregation of duties, i.e., the determination of incompatible duties, access, or management override and its effect on controls; (3) electronic data processing (EDP) factors, i.e., the importance of the computer to the processing and control of data, and relating how the lack of specific general computer controls will affect the various application programs; and (4) the adequacy of the working papers, i.e., how well those preparing the workpapers have captured and documented all relevant aspects of the client's accounting system in order to draw control conclusions.

The data cues are analyzed from each of the above perspectives, but then must be integrated in deciding which controls may be effective, which controls should be compliance tested, and what type of compliance tests should be performed and how large of a sample would be sufficient. Our primary subject has reported flipping from one view to another in his mind as he analyzes the data and how a change in one view affects his other views of the data. Much of this "flipping from one view to another" shows up in the protocol. The graph in Figure 15 illustrates that there may be mention of more than one reference frame in any of the protocol units. Also note that the primary frame of reference is processing, which enjoys more sustained attention. It should be noted that the working papers are organ. ized more from this frame of reference. 


\subsection{Cognitive Processes}

Cognitive process decision operators provide the links between individual knowledge states at the micro level. Scoring the protocol for cognitive processes allows the determination of which operators are used by auditors in their evaluation of internal controls. Figure 16 provides a list of operators scored.

The use of the data search operator is consistent with our previous observation that auditors perform a comprehensive review of all working papers available. In addition, we find that auditors deal with uncertainty by raising questions, building conjectures, making assumptions, and proposing numerous tentative evaluations. This use of discrete assumed outcomes rather than probabilistic assessments of uncertainty is consistent with Biggs et al. [1985] and Doyle's [1983a,b] "reasoning by assumption." No probabilistic remark, such as "I'm 75 percent positive that invoices are adequately accounted for," was found in any of the protocols.

The micro analysis, scoring of the cognitive processes, helped most in the model building process when scored for within the bounds of the specific goals and objectives that direct the search and confirmation processes. The macro analysis of the protocol provided the control structure around which the model was built.

\subsection{Conclusions from the Research}

A primary contribution of this study arises from our limited understanding of the processes auditors use in reviewing and evaluating internal accounting controls. Analyzing and modelling the knowledge states and related reasoning processes that managers and partners use help to describe what it takes to have expertise in auditing. The research facilitates transmission, reproduction, consensus, enhancement, automation of the routine aspects of the expert's task, and help experts and novices better solve problems [Hayes-Roth, Waterman and Lenat, 1983]. 
COGNITIVE PROCESSES

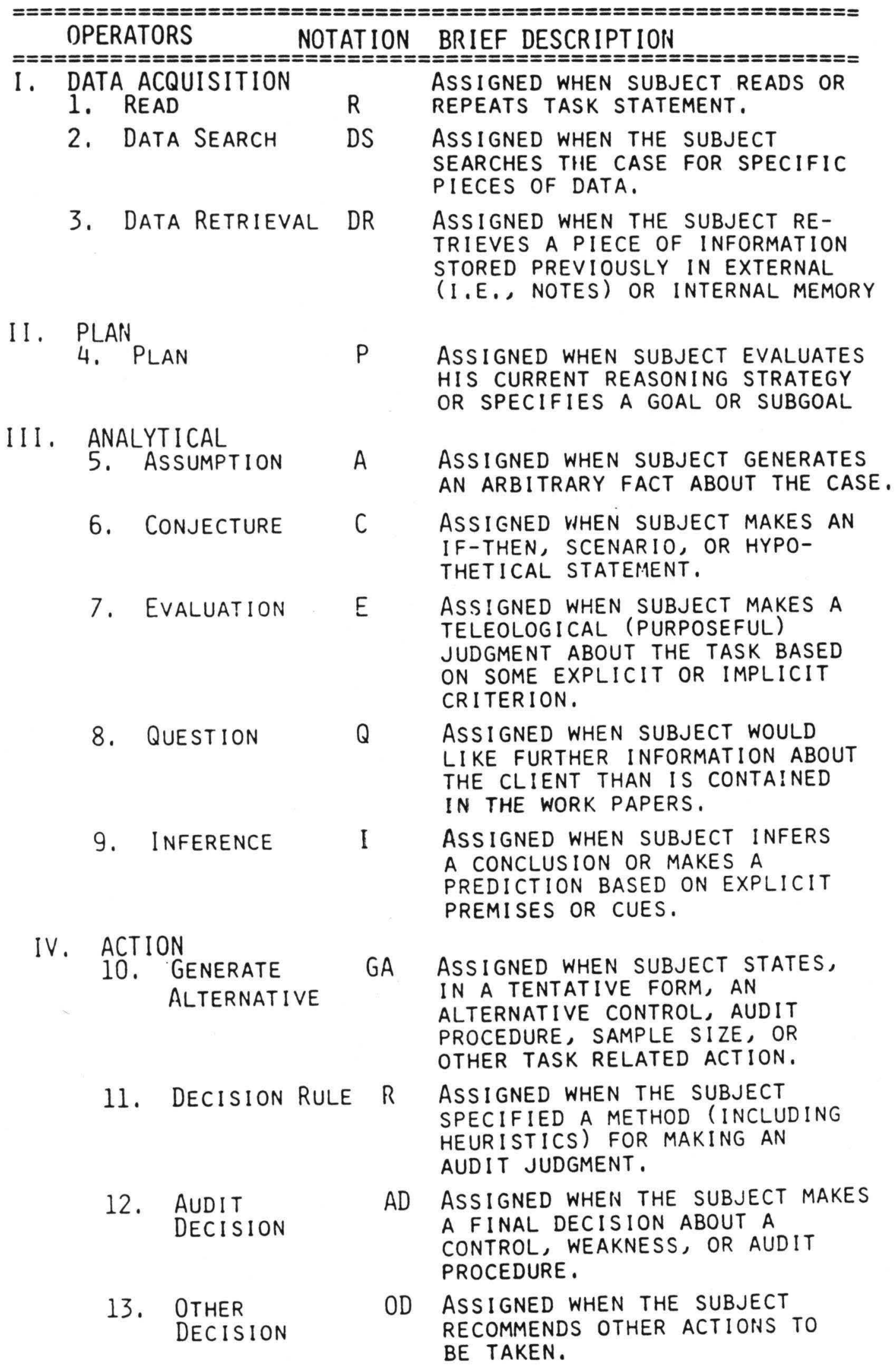

(See Malone [1984] and Biggs et al. [1985].)

Figure 16 


\section{CONCLUSIONS AND SUGGESTIONS FOR FUTURE RESEARCH}

The objective of this research is to formulate and test a model of the processes employed by expert auditors in reviewing and evaluating internal accounting controls. The purpose of the research is to describe in greater detail the processes that are used in auditor judgements. The computational model provides a means of testing the understanding.

A primary contribution of this study arises from our limited understanding of the processes auditors use in reviewing and evaluating internal accounting controls. Analyzing and modelling the knowledge states and related reasoning processes that managers and partners use helps to describe what it takes to have expertise in auditing. This research facilitates transmission, reproduction, consensus, enhancement, automation of the routine aspects of the expert's task, and help experts and novices better solve problems [Hayes-Roth, Waterman and Lenat, 1983].

In future research extensions, we plan to join the TICOM modelling, querying, and evaluation techniques under the control of an expert system decision model. Such a marriage could allow the resulting system to query the user for appropriate modelling information, represent the client's system as a TICOM model, then deciding what questions would be interesting to ask, query the model and combine the results into a recommendation of controls to test and a report on control weaknesses. The resulting system would allow both individual companies and auditors to make effective and efficient evaluations of internal controls.

Other research extensions of the current research include extending the cognitive simulation approach developed in this model to other auditing tasks. The possibilities are numerous. Examples of such tasks include combining the results of the compliance tests into a final evaluation of internal control and then planning which substantive test to perform.

Future research will also include such sophistication in auditing as to allow one computer system to analyze another computer's data base system and automatically pick and help evaluate audit samples. There is no lack of interesting research issues 
involving the use of DSS/AI/ES systems in auditing.

\section{REFERENCES}

Alter, S. (1977). "A Taxonomy of Decision Support Systems," Sloan Management Review, 19, 1, Fall, pp. 39-56.

Arens, A.A. and Loebbecke, J.K. (1984). Auditing: An Integrated Approach, 3rd Edition, Prentice-Hall, Inc., Englewood Cliffs, N.J.

American Institute of Certified Public Accountants (1972). Statement on Auditing Standards, AU Section 320, November.

Barr, A, and Feigenbaum, E.A. (1981). The Handbook of Artificial Intelligence, 1 , HeurisTech Press.

Bailey, A.D. Jr., Duke, G.L., Johnson, P.E., Meservy, R.D. and Thompson, W. (1984). "Auditing Internal Controls: A Computational Model of the Review Process," research proposal, University of Minnesota.

Bailey, A.D. Jr., Duke, G.L., Gerlach, J., Ko, C., Meservy, R.D. and Whinston, A.B. (1985). "TICOM and the Analysis of Internal Controls," The Accounting Review, April.

Bailey, A.D. Jr., Gerlach, J.H., McAffee, R.P. and Whinston, A.B. (1981). "An Application of Complexity Theory to the Analys is of Internal Control Systems," Auditing: A Journal of Practice and Theory, Summer.

Bailey, A.D. Jr., Gerlach, J.H., McAfee, R.P., Whinston, A.B. and Watson, D.J.H. (1983). "OIS Technology and Accounting: Partners in Conflict," in Data Base Management: Theory and Applications, C. Holsapple and A. Whinston (eds.), NATO Advanced Study Institute Series, D. Reidel Publishing Co., London.

Balachandran, B.V. and Zoltners, A.A. (1981). "An Interactive Audit-Staff Scheduling Decision Support System," The Accounting Review, Uctober.

Biggs, S.F. (1984). "Empirical Analysis and Expert System Development for Auditors Going-Concern Judgment," Symposium on Decision Support Systems in Auditing, University of Southern California.

Biggs, S.F., Messier, W.F. Jr. and Hansen, J.V. (1985). "A Study of the Predecisional Behavior of Computer Audit Specialists in Advanced EDP Environments," ARC Working Paper No. $84-1$.

Biggs, S.F. and Mock, T.J. (1983). "An Investigation of Auditor Decision Processes in the Evaluation of Internal Controls and Audit Scope Decisions," Journal of Accounting Research, Spring. 
Braun, H.M. and Chandler, J.S. (1982). "Development of an Expert System to Assist Auditors in the Investigation of Analytical Review Fluctuations," research proposal, University of Illinois.

Cohen, J. (1960). "A Coefficient of Agreement for Nominal Scales," Educational and Psychological Measurement, 26 , pp. $37-46$.

Davis, R. (1982). "Expert Systems: Where Are We? And Where Do We Go From Here?," The AI Magazine, Spring.

Doyle, J. (1983b). "Some Theories of Reasoned Assumptions: An Essay in Rational Psychology," Working Paper CS-83-125, Computer Science Department, Carnegie-Mellon University, May.

Dungan, C. and Chandler, J. (1980). "Development of KnowledgeBased Expert Systems to Model Auditors' Decision Processes," unpublished paper, University of Illinois.

Einhorn, H.J. (1980). "Learning From Experience and Suboptimal Rules in Decision Making," in Cognitive Processes in Choice and Decision Behavior, T.S. Wallsten (ed.), Erlbaum, Hillsdale, N.J.

Elstein, A.S., Shulman, A.S. and Sprafka, S.M. (1978). Medical Problem Solving: An Analysis of Clinical Reasoning, Harvard University Press, Cambridge, MA.

Ericsson, K.A. and Simon, H.A. (1984). Protocol Analysis: Verbal Reports as Data, The MIT Press, Cambridge, MA.

Feigenbaum, E.A. (1978). "The Art of Artificial Intelligence -Themes and Case Studies of Knowledge Engineering," Proceedings of the National Computer Conference.

Hansen, J.V. and Messier, W.F. (1982). "Expert Systems for Decision Support in EDP Auditing," International Journal of Computer and Information Sciences, October.

Hayes-Roth, F., Waterman, D.A. and Lenat, D.B. (1983). Building Expert Systems, Addison-Wesley.

Johnson, K.P. and Jaenicke, H.R. (1980). Evaluating Internal Control, John Wiley \& Sons.

Johnson, P.E. (1983). "The Expert Mind: A New Challenge for the Information Scientist."

Johnson, P.E. (1983). "What Kind of Expert Should a System Be?," The Journal of Medicine and Philosophy, pp. 77-97.

Johnson, P.E., Hassebrock, F., Duran, A.S. and Mollar, J. (1982). "Multimethod Study of Clinical Judgment," Organizational Behavior and Human Performance, 30, pp. 201-230. 
Joyce, E.J. and Libby, R. (1982). "Behavioral Studies of Audit Decision Making," Journal of Accounting Literature.

Kleinmuntz, B. (1968). "The Process of Clinical Information by Man and Machine," in Formal Representations of Human Judgment, B. Kleinmuntz (ed.), John Wiley and Sons, New York.

Libby, R. (1981). Accounting and Human Information Processing: Theory and Applications, Prentice-Hali.

Lindsay, R.K., Buchanan, B.G., Feigenbaum, E.A. and Lederberg, J. (1980). "Applications of Artificial Intelligence for Organic Chemistry: The DENDRAL Project," McGraw-Hill.

Loebbecke, J.K. and Zuber, G.R. (1980). "Evaluating Internal Controls," The Journal of Accountancy, February.

Mair, W.C., Wood, D.R. and Davis, K.W. (1978). Computer Control \& Audit, The Institute of Internal Auditors, Touche Ross \& Co., Florida, p. 34.

Mautz, R. and Winjum, J. (1981). "Criteria for Management Control Systems," Financial Executive Research Institute.

Melone, N.P. (1984). "A Strategy for Knowledge-Based Decisior. Support: Decision Making Expertise in Corporate Acquisitions," Unpublished Ph.D. Dissertation Proposal, University of Minnesota.

McCarty (1977). "Reflexions on Taxman: An Experiment in Artificial Intelligence and Legal Reasoning," Harvard Law Review, March, pp. 837-893.

Michaelson, R.H. (1982). "An Expert System for Federal Tax Planning," Working Paper, University of Nebraska.

Mock, T. and Vertinsky, I. (1984). "DSS-RAA: Design Highlights," Symposium on Decision Support Systems for Auditing, University of Southern California.

Moen, James B. (1984). "Algorithms and Data Structures in Galen," Working Paper, University of Minnesota.

Mutchler, J.F. (1984). "Alditors' Perceptions of the Going Concern Opinion Decision," Auditing: A Journal of Practice and Theory, Spring, pp. 17-30.

Nesbett, R.E. and Wilson, T.D. (1977). "Telling More Than We Can Know: Verbal Reports On Mental Processes," Psychological Review, May.

Newel1, A. and Simon, H.A. (1972). Human Problem Solving, Prentice-Hall.

Peat, Marwick, Mitchell \& Co. (1986). Research Opportunities in Auditing, New York. 
Shortliffe, E.H. (1976). "Computer-Based Medical Consultations: MYCIN," North-Holland.

Stefik, M., Aikins, J., Balzer, R., Benoit, J., Birnbaum, L., Hayes-Roth, F. and Sacerdoti, E. (1982). The Organization of Expert Systems: A Prescriptive Tutorial, Xerox Palo Alto Research Centers.

Thompson, W.B., Johnson, P.E. and Moen, J.B. (1983). "Recognition-Based Diagnostic Reasoning," IJCAI Proceedings,

van Melle, W., Shortliffe, E.H., and Buchanan, B.G. (1981). "EMYCIN: A Domain-Independent System that Aids in Constructing Knowledge-Based Consultation Programs," Machine Intelligence, Infotech State of the Art Report, 9 , 3 .

Weber, R. (1982). EDP Auditing: Conceptual Foundations and Practice, McGraw-Hill, New York. 